\title{
Semidefinite Programming for Approximate Maximum Likelihood Sinusoidal Parameter Estimation
}

\author{
Kenneth W. K. Lui (EURASIP Member) and H. C. So \\ Department of Electronic Engineering, City University of Hong Kong, Tat Chee Avenue, Kowloon, Hong Kong \\ Correspondence should be addressed to H. C.So, hcso@ee.cityu.edu.hk
}

Received 19 February 2009; Revised 8 September 2009; Accepted 20 November 2009

Recommended by T.-H. Li

\begin{abstract}
We study the convex optimization approach for parameter estimation of several sinusoidal models, namely, single complex/real tone, multiple complex sinusoids, and single two-dimensional complex tone, in the presence of additive Gaussian noise. The major difficulty for optimally determining the parameters is that the corresponding maximum likelihood (ML) estimators involve finding the global minimum or maximum of multimodal cost functions because the frequencies are nonlinear in the observed signals. By relaxing the nonconvex ML formulations using semidefinite programs, high-fidelity approximate solutions are obtained in a globally optimum fashion. Computer simulations are included to contrast the estimation performance of the proposed semidefinite relaxation methods with the iterative quadratic maximum likelihood technique as well as Cramér-Rao lower bound.
\end{abstract}

Copyright (C) 2009 K. W. K. Lui and H. C. So. This is an open access article distributed under the Creative Commons Attribution License, which permits unrestricted use, distribution, and reproduction in any medium, provided the original work is properly cited.

\section{Introduction}

The problem of sinusoidal parameter estimation in additive noise has been an important research topic because of its numerous applications in science and engineering [1-5]. The typical parameters of interest are frequencies, amplitudes, and phases. As the frequencies are nonlinear functions in the received noisy measurements, they are usually estimated prior to the amplitude and phase estimation. In this paper, we focus on optimal parameter estimation for the representative signal models of single complex/real tone, multiple complex sinusoids, and single two-dimensional (2D) complex tone, although the development can also be extended to other related models [6-13] in the literature.

Utilizing the linear prediction polynomial (LPP) of the noise-free signal, an ML estimator for sinusoidal parameter estimation has been proposed in $[7,8]$ under the standard assumption of Gaussian noise. The nonlinear least squares (NLSs) estimator [14] will also produce ML estimation performance when the noise is white. In particular, the ML frequency estimate of a single complex sinusoid in white Gaussian noise can be obtained from the periodogram peak $[2,15]$. However, the cost functions of all these ML methods are multimodal and two steps are typically involved in the estimation procedure. First suboptimal initial parameter estimates are obtained by discrete Fourier transform (DFT), linear prediction [16], or other means [7, 8]. A refinement is then made through an iterative optimization of the cost functions. As a result, this means that sufficiently accurate initial estimates are necessary for global convergence. In this work, we approximate the nonconvex ML formulations as convex optimization problems so that a high-quality global solution is guaranteed. In fact, convex optimization [1719] has been applied in a number of signal processing and communication applications [20-28]. Phase-shift keying demodulation, which is a Boolean quadratic problem with polynomial constraints, is studied in [20]. Multiple-inputmultiple-output detection, which is a closet lattice point search problem, is examined in [21-23]. In addition, the problems of multigroup multicast transmit beamforming, digital filter design and localization are considered in [2428] respectively. These problems are nonconvex in nature but can be approximated as constrained convex optimization programs where the objective functions are convex while the inequality constraints are convex and the equality constraints are linear.

The rest of the paper is organized as follows. We start with the simplest single complex tone model corrupted by 
white Gaussian noise in Section 2. Through semidefinite relaxation (SDR), the periodogram, LPP and NLS estimators are approximated as convex programs. In the first two SDR schemes, the frequency parameter is obtained prior to amplitude and phase estimation while the third one is a waveform estimator. In Section 3, the SDR approximations of the LPP and NLS estimators for a noisy real sinusoid are derived. Section 4 extends the SDR-LPP methodology in Sections 2 and 3 to the multiple tone model. An illustration for the dual tone case is provided. The SDR formulations of the periodogram and NLS estimators for a 2D complex tone are derived in Section 5, while the LPP version is omitted as its development is too tedious. Fast implementation of some developed SDR solutions for large observation records is suggested in Section 6. In Section 7, numerical examples are included to contrast the estimation performance of the proposed SDR methods with the iterative quadratic maximum likelihood (IQML) technique $[7,8]$ as well as Cramér-Rao lower bound (CRLB). It is worthy to point out that although the algorithms can work for noises which are non-Gaussian and/or colored, we keep the assumption of white Gaussian noise so that their optimality can be easily assessed by comparing with the benchmark of CRLB. Finally, conclusion is drawn in Section 8. A list of symbols that are used in the paper is given in Table 1.

\section{Single Complex Tone}

In this section, we tackle the simplest form of the sinusoidal estimation problem, namely, finding the parameters of a complex tone in additive noise. The discrete-time signal model is

$$
y(n)=s(n)+\eta(n), \quad n=1,2, \ldots, N
$$

where

$$
s(n)=A \exp \{j(\omega n+\phi)\} .
$$

The $A>0, \omega \in(-\pi, \pi)$ and $\phi \in[0,2 \pi)$ are deterministic unknown constants which denote the sinusoidal amplitude, frequency, and phase, respectively, while the additive noise $\eta(n)$ is a zero-mean complex Gaussian process. It is assumed that the covariance matrix of the noise vector $\boldsymbol{\eta}=[\eta(1), \eta(2), \ldots, \eta(N)]^{T}$, denoted by $\boldsymbol{\Sigma}_{\eta}=E\left\{\boldsymbol{\eta} \boldsymbol{\eta}^{H}\right\}$, where $E$ is the expectation operator, is known up to a scalar. Our objective is to estimate $A, \omega$, and $\phi$ from the $N$ noisy measurements of $y(n)$. Three SDR methods namely, periodogram, PPL, and NLS, are proposed to perform the estimation as follows.

2.1. Periodogram Approach. In this subsection, we assume that $\{\eta(n)\}$ are independent and identically distributed (IID), that is, $\boldsymbol{\Sigma}_{\eta}=\sigma_{\eta}^{2} \mathbf{I}_{N}$ where $\sigma_{\eta}^{2}$ is unknown. In this case, the ML frequency estimate can be obtained from the periodogram maximum [15]:

$$
\max _{\omega}|y(\omega)|^{2}
$$

where $y(\omega)=\sum_{n=1}^{N} y(n) \exp \{-j \omega n\}$ is the discrete-time Fourier transform (DTFT) of $y(n)$. The difficulty of solving (3) lies in its multimodality. A conventional strategy is first to obtain an initial estimate from the DFT peak and then apply a gradient search for (3). Here, (3) will be relaxed as a convex optimization problem so that a global high-quality solution is guaranteed. First, it is clear that (3) is equivalent to the following constrained problem:

$$
\begin{aligned}
& \max _{\mathbf{c}, \omega}\left|\mathbf{c}^{H} \mathbf{y}\right|^{2} \\
& \text { s.t. } \quad c(n)=\exp \{j \omega n\}, \quad n=1,2, \ldots, N,
\end{aligned}
$$

where $\mathbf{y}=[y(1), y(2), \ldots, y(N)]^{T}$ and $\mathbf{c}=$ $[c(1), c(2), \ldots, c(N)]^{T}$. The constraints make (4) a nonconvex program as $\exp \{\cdot\}$ is a periodic function. Furthermore, if the constraints are removed, maximization of the convex objective function $\left|\mathbf{c}^{H} \mathbf{y}\right|^{2}=\mathbf{y}^{H} \mathbf{c c}^{H} \mathbf{y}$ will result in $c(n) \rightarrow \infty$ as the function is not bounded above. To avoid this, we define $\mathbf{C}=\mathrm{cc}^{H} \in \mathbb{C}^{N}$ to make the objective function affine and now $\left|\mathbf{c}^{H} \mathbf{y}\right|^{2}$ is rewritten as $\mathbf{y}^{H} \mathbf{C y}$ which is linear in C. Using the sinusoidal property of $c(n)$, it is easy to show that $\mathbf{C}$ has a special structure of the form:

$$
\begin{aligned}
\mathbf{C} & =\left[\begin{array}{cccc}
1 & c(1)^{*} & \cdots & c(N-1)^{*} \\
c(1) & 1 & \cdots & c(N-2)^{*} \\
\vdots & \vdots & \ddots & \vdots \\
c(N-1) & c(N-2) & \cdots & 1
\end{array}\right] \\
& =\operatorname{Toeplitz}\left(\left[1, c(1)^{*}, c(2)^{*}, \ldots, c(N-1)^{*}\right]\right) .
\end{aligned}
$$

Then (4) is equivalent to

$$
\max _{\mathbf{C}, \mathbf{c}} \mathbf{y}^{H} \mathbf{C y}
$$

s.t. $\quad \mathbf{C}=\mathbf{c c}^{H}=\operatorname{Toeplitz}\left(\left[1, c(1)^{*}, c(2)^{*}, \ldots, c(N-1)^{*}\right]\right)$

Finally, relaxing $\mathbf{C}=\mathbf{c c}^{H}$ to $\mathbf{C} \succeq \mathbf{c c}^{H}$ yields the SDR formulation for single complex tone frequency estimation:

$$
\begin{aligned}
\max _{\mathbf{C}, \mathbf{c}} & \mathbf{y}^{H} \mathbf{C y} \\
\text { s.t. } & {\left[\begin{array}{cc}
\mathbf{C} & \mathbf{c} \\
\mathbf{c}^{H} & 1
\end{array}\right] \succeq \mathbf{0}_{N+1}, } \\
& \mathbf{C}=\operatorname{Toeplitz}\left(\left[1, c(1)^{*}, c(2)^{*}, \ldots, c(N-1)^{*}\right]\right) .
\end{aligned}
$$

Note that no constraints are imposed on $c(n), n=$ $1,2, \ldots, N-1$. In the optimization literature, there are readily available solvers for finding the globally optimum solution for (7), such as SeDuMi [29] and SDPT3 [30, 31], and they basically employ the interior-point methods [32, 33]. As the estimate of $\mathbf{c}$, namely, $\hat{\mathbf{c}}$, is constrained as a unity-amplitude tone with zero initial phase, the frequency estimate, denoted by $\hat{\omega}$, is computed as

$$
\widehat{\omega}=\angle \widehat{c}(1)
$$


TABLE 1: List of symbols.

\begin{tabular}{|c|c|}
\hline Symbol & Meaning \\
\hline $\mathbb{R}^{N}$ & Set of $N \times N$ real matrices \\
\hline $\mathbb{C}^{N}$ & Set of $N \times N$ complex matrices \\
\hline$[\mathbf{a}]_{i}$ & $i$ th element of vector $\mathbf{a}$ \\
\hline$[\mathbf{A}]_{i, j}$ & $(i, j)$ entry of matrix $\mathbf{A}$ \\
\hline $\mathbf{A}^{T}$ & Transpose of $\mathbf{A}$ \\
\hline $\mathbf{A}^{H}$ & Hermitian transpose of $\mathbf{A}$ \\
\hline $\mathbf{A}^{*}$ & Conjugate of $\mathbf{A}$ \\
\hline $\operatorname{tr}(\mathbf{A})$ & Trace of $\mathbf{A}$ \\
\hline $\mathbf{A} \succeq \mathbf{B}$ & $\mathbf{A}-\mathbf{B}$ is positive semidefinite \\
\hline$\angle(a)$ & Angle of complex variable $a$ \\
\hline $\mathbf{1}_{N}$ & Square matrix with size $N \times N$ of 1 \\
\hline $\mathbf{0}_{N}$ & Square matrix with size $N \times N$ of 0 \\
\hline $\mathbf{I}_{N}$ & Identity matrix with size $N \times N$ \\
\hline $\operatorname{diag}(\mathbf{a})$ & Diagonal matrix with vector $\mathbf{a}$ as main diagonal \\
\hline $\operatorname{diag}(\mathbf{A}, k)$ & Column vector formed by the elements of $k$ th diagonal of $\mathbf{A}$ \\
\hline $\operatorname{blkdiag}\left(\mathbf{A}_{1}, \mathbf{A}_{2}, \ldots, \mathbf{A}_{n}\right)$ & Block diagonal matrix with its diagonal elements are square matrices of $\mathbf{A}_{1}, \mathbf{A}_{2}, \ldots, \mathbf{A}_{n}$ \\
\hline Toeplitz(a) & Symmetric or Hermitian Toeplitz matrix formed by a, which is the first row \\
\hline$\widehat{\mathbf{A}}$ & Estimate of $\mathbf{A}$ \\
\hline$E\{\mathbf{A}\}$ & Expectation of $\mathbf{A}$ \\
\hline$\lfloor\mathbf{A}\rfloor$ & Round elements of $\mathbf{A}$ to the nearest integers towards $-\infty$ \\
\hline $\operatorname{sign}(\mathbf{A})$ & Sign of $\mathbf{A}$ \\
\hline $\operatorname{vec}(\mathbf{A})$ & Vectorization of A to produce a column vector containing all its elements \\
\hline \multirow[t]{3}{*}{$\operatorname{perm}_{1}(\mathbf{A}, M, N) \in \mathbb{C}^{M N}$} & {$\left[\operatorname{perm}_{1}(\mathbf{A}, M, N)\right]_{k, l}=[\mathbf{A}]_{i, j}, i, j=1,2, \ldots, M N$} \\
\hline & $k=M(i-1)-(M N-1)\lfloor i / N\rfloor+1$ \\
\hline & $l=M(j-1)-(M N-1)\lfloor j / N\rfloor+1($ see the appendix $)$ \\
\hline \multirow[t]{2}{*}{$\operatorname{perm}_{2}(\mathbf{A}, M, N) \in \mathbb{C}^{\min ([M, N])}$, } & {$\left[\operatorname{perm}_{2}(\mathbf{A}, M, N)\right]_{k, l}=[\mathbf{A}]_{i, j}, i, j=1,2, \ldots, M N$} \\
\hline & $i=M(k-1)+k, j=M(l-1)+l($ see the appendix $)$ \\
\hline $\min (\mathbf{a})$ & Minimum element of vector a \\
\hline
\end{tabular}

With the use of (1), (2), and $\widehat{c}$, the amplitude and phase estimates, denoted by $\hat{A}$ and $\hat{\phi}$, are calculated using least squares (LS) as

$$
\begin{gathered}
\hat{A}=\frac{\left|\hat{\mathbf{c}}^{H} \mathbf{y}\right|}{N}, \\
\hat{\phi}=-\angle\left(\hat{\mathbf{c}}^{H} \mathbf{y}\right) .
\end{gathered}
$$

2.2. Linear Prediction Polynomial Approach. Following the development in [34] with the use of the property of $s(n)=$ $\exp \{j \omega\} s(n-1)$, it is shown that the ML frequency estimator in the presence of Gaussian noise can be determined from minimization of the following multimodal function:

$$
\min _{c(1)}\left(\mathbf{y}_{2}-c(1) \mathbf{y}_{1}\right)^{H} \boldsymbol{\Sigma}_{e}^{-1}\left(\mathbf{y}_{2}-c(1) \mathbf{y}_{1}\right)
$$

where $\mathbf{y}_{i}=[y(i), y(2+i), \ldots, y(N-2+i)]^{T}, i=1,2$, and $\boldsymbol{\Sigma}_{e}=E\left\{\mathbf{e e}^{H}\right\} \in \mathbb{C}^{N-1}$ is a weighting matrix with $\mathbf{e}=$ $[e(1), e(2), \ldots, e(N-1)]^{T}$ whose element is $e(n)=\eta(n+$ 1) $-c(1) \eta(n), n=1,2, \ldots, N-1$, and the presence of $c(1)$ in $\boldsymbol{\Sigma}_{e}^{-1}$ makes (11) violating the convex framework. We refer the estimator of (11) to as LPP approach. Unlike the periodogram, the ML property of (11) holds for a general form of $\boldsymbol{\Sigma}_{\eta}$. Moreover, (11) can be extended to the scenarios of multiple real or complex sinusoids. It is noteworthy that the iterative quadratic maximum likelihood (IQML) [7] technique provides a standard solution to (11) by relaxing the LPP cost function to a quadratic form, although a sufficiently close initial guess, which is usually obtained through solving (11) with $\boldsymbol{\Sigma}_{e}=\mathbf{I}_{N-1}$, is required to attain the global minimum. Furthermore, the LPP scheme has similarities to the linear filtering approach in $[35,36]$.

For simplicity but without loss of generality, here we assume that $\boldsymbol{\Sigma}_{\eta}=\sigma_{\eta}^{2} \mathbf{I}$ and $\boldsymbol{\Sigma}_{e}$ is then of the form:

$$
\boldsymbol{\Sigma}_{e}=\text { Toeplitz }([2,-c(1) * \overbrace{0,0, \ldots, 0}^{N-3}]) \sigma_{\eta}^{2} .
$$


Ignoring the scaling factor of $\sigma_{\eta}^{2}$ in (12), the minimizer of (11) is equivalent to

$$
\begin{array}{ll}
\min _{v, c(1)} & v \\
\text { s.t. } & v=\left(\mathbf{y}_{2}-c(1) \mathbf{y}_{1}\right)^{H} \mathbf{\Sigma}_{e}^{-1}\left(\mathbf{y}_{2}-c(1) \mathbf{y}_{1}\right), \\
& \mathbf{\Sigma}_{e}=\text { Toeplitz }\left([2,-c(1)^{*}, \overbrace{0,0, \ldots, 0}^{N-3}]\right) .
\end{array}
$$

By replacing (14) with Schur complement and relax the resultant expression, the SDR formulation for solving $c(1)$ is

$$
\begin{array}{ll}
\min _{v, c(1)} & v \\
\text { s.t. } & {\left[\begin{array}{cc}
v & \left(\mathbf{y}_{2}-c(1) \mathbf{y}_{1}\right)^{H} \\
\left(\mathbf{y}_{2}-c(1) \mathbf{y}_{1}\right) & \mathbf{\Sigma}_{e}
\end{array}\right] \geq \mathbf{0}_{N},} \\
& \mathbf{\Sigma}_{e}=\text { Toeplitz }\left([2,-c(1)^{*}, \overbrace{0,0, \ldots, 0}^{N-3}]\right) .
\end{array}
$$

It is noteworthy that the inverse operator is removed in the Schur complement and there is a linear relation between the weighting matrix elements and the parameter estimate. As a result, tight constraints are formed automatically. After getting $\widehat{c}(1)$, the estimates of frequency, amplitude and phase are computed using (8), (9), and (10), respectively.

2.3. Nonlinear Least Squares Approach. It is straightforward to see that the NLS estimator for the sinusoidal parameters can be expressed as the following constrained optimization problem:

$$
\begin{aligned}
& \min _{\mathbf{s}, A, \omega, \phi}(\mathbf{y}-\mathbf{s})^{H} \boldsymbol{\Sigma}_{\eta}^{-1}(\mathbf{y}-\mathbf{s}) \\
& \text { s.t. } \quad s(n)=A \exp \{j(\omega n+\phi)\}, \quad n=1,2, \ldots, N,
\end{aligned}
$$

where $\mathbf{y}=[y(1), y(2), \ldots, y(N)]^{T}$ and $\mathbf{s}=[s(1), s(2)$, $\ldots, s(N)]^{T}$. Similar to (4), the constraints, $s(n)=A \exp \{j \omega+$ $\phi\}, n=1,2, \ldots, N$, make (17) violating the convex framework. Based on the linear prediction property, the constraint of $s(n)$ can be alternatively expressed as

$$
\begin{array}{r}
s(n)-\exp \{j \omega m\} s(n-m)=0 \\
\Longrightarrow s(n) s(n-m)^{*}=A^{2} \exp (j \omega n)=A^{2} c(m), \\
\\
m=1,2, \ldots, N-1 .
\end{array}
$$

Defining $\mathbf{S}=\mathbf{s s}^{H} \in \mathbb{C}^{N}$ with $[\mathbf{S}]_{i, j}=s(i) s(j)^{*}=A^{2} c(i-j)$ and using (18), the constraint in (17) is equivalent to

$$
\begin{aligned}
\mathbf{S} & =\mathbf{s s}^{H} \\
& =\left[\begin{array}{cccc}
A^{2} c(0) & A^{2} c(1)^{*} & \cdots & A^{2} c(N-1)^{*} \\
A^{2} c(1) & A^{2} c(0) & \cdots & A^{2} c(N-2)^{*} \\
\vdots & \vdots & \ddots & \vdots \\
A^{2} c(N-1) & A^{2} c(N-2) & \cdots & A^{2} c(0)
\end{array}\right] \\
& =\text { Toeplitz }\left(\left[A^{2} c(0), A^{2} c(1)^{*}, \ldots, A^{2} c(N-1)^{*}\right]\right) .
\end{aligned}
$$

On the other hand, expanding the objective function of (17) yields

$$
\mathbf{s}^{H} \boldsymbol{\Sigma}_{\eta}^{-1} \mathbf{s}-\mathbf{y}^{H} \boldsymbol{\Sigma}_{\eta}^{-1} \mathbf{s}-\mathbf{s}^{H} \boldsymbol{\Sigma}_{\eta}^{-1} \mathbf{y}+\mathbf{y}^{H} \boldsymbol{\Sigma}_{\eta}^{-1} \mathbf{y} .
$$

We drop the term $\mathbf{y}^{H} \boldsymbol{\Sigma}_{\eta}^{-1} \mathbf{y}$ which takes no effect in the minimization and replace $\mathbf{s}^{H} \boldsymbol{\Sigma}_{\eta}^{-1} \mathbf{s}$ with $\operatorname{tr}\left(\boldsymbol{\Sigma}_{\eta}^{-1} \mathbf{S}\right)$. Relaxing $\mathbf{S}=\mathbf{s s}^{H}$ as $\mathbf{S} \succeq \mathbf{s s}^{H}$, the SDR formulation becomes

$$
\begin{array}{ll}
\min _{\mathbf{S}, \mathbf{s}} & \operatorname{tr}\left(\boldsymbol{\Sigma}_{\eta}^{-1} \mathbf{S}\right)-\mathbf{y}^{H} \boldsymbol{\Sigma}_{\eta}^{-1} \mathbf{s}-\mathbf{s}^{H} \boldsymbol{\Sigma}_{\eta}^{-1} \mathbf{y} \\
\text { s.t. } & {\left[\begin{array}{cr}
\mathbf{S} & \mathbf{s} \\
\mathbf{s}^{H} & 1
\end{array}\right] \succeq \mathbf{0}_{N+1},} \\
& \quad \mathbf{S}=\operatorname{Toeplitz}\left(\left[A^{2} c(0), A^{2} c(1)^{*}, \ldots, A^{2} c(N-1)^{*}\right]\right) .
\end{array}
$$

Note that no constraints are imposed on $A^{2} c(n), n=$ $0,1, \ldots, N-1$, and the SDR solution, namely, $\hat{\mathbf{s}}$, will be the estimated waveform which is a pure complex tone. The parameter estimates are easily computed as

$$
\begin{gathered}
\hat{\omega}=\angle[\hat{\mathbf{S}}]_{2,1}, \\
\hat{A}=\sqrt{[\hat{\mathbf{S}}]_{1,1}} \\
\hat{\phi}=\angle\left(\hat{\boldsymbol{s}}(1)[\hat{\mathbf{S}}]_{1,2}\right) .
\end{gathered}
$$

\section{Single Real Tone}

In this section, we switch to find the parameters of a noisy real tone which is another fundamental sinusoidal estimation problem. The signal model is

$$
x(n)=s(n)+q(n), \quad n=1,2, \ldots, N,
$$

where

$$
s(n)=A \cos (\omega n+\phi) .
$$

The real and complex models are very similar except now $\omega \in(0, \pi)$ and the Gaussian noise $q(n)$ is real. Similarly, the covariance matrix of the noise vector $\mathbf{q}=$ $[q(1), \mathrm{q}(2), \ldots, q(N)]^{T}$, denoted by $\boldsymbol{\Sigma}_{q}=E\left\{\mathbf{q q}^{T}\right\}$, is assumed known up to a scalar. As the periodogram is not an optimum estimator for a real tone, only the LPP and NLS approaches will be presented as follows. 
3.1. Linear Prediction Polynomial Approach. Let $\rho_{1}=\cos (\omega)$. Utilizing $s(n)-2 \rho_{1} s(n-1)+s(n-2)$ and [34], the LPP estimator for $\rho_{1}$ is

$$
\min _{\rho_{1}}\left(\mathbf{x}_{3}-2 \rho_{1} \mathbf{x}_{1}+\mathbf{x}_{2}\right)^{T} \boldsymbol{\Sigma}_{e}^{-1}\left(\mathbf{x}_{3}-2 \rho_{1} \mathbf{x}_{1}+\mathbf{x}_{2}\right)
$$

where $\mathbf{x}_{i}=[x(i), x(i+1), \ldots, x(N+3-i)]^{T}, i=1,2,3$, and $\boldsymbol{\Sigma}_{e}=E\left\{\mathbf{e e}^{T}\right\} \in \mathbb{R}^{N-2}$ with $\mathbf{e}=[e(1), e(2), \ldots, e(N-2)]^{T}$ whose element is $e(n)=q(n+2)-2 \rho_{1} q(n+1)+q(n), n=$ $1,2, \ldots, N-2$.

Assuming that $\boldsymbol{\Sigma}_{q}=\sigma_{q}^{2} \mathbf{I}_{N}$, a scaled version of $\boldsymbol{\Sigma}_{e}$ is employed:

$$
\boldsymbol{\Sigma}_{e}=\text { Toeplitz }\left([2+4 \rho_{1}^{2},-4 \rho_{1}, 1, \overbrace{0, \ldots, 0}^{N-5}]^{T}\right) .
$$

The minimizer of (27) is identical to

$$
\begin{array}{ll}
\min _{v, \rho_{1}} & v \\
\text { s.t. } & v=\left(\mathbf{x}_{3}-2 \rho_{1} \mathbf{x}_{1}+\mathbf{x}_{2}\right)^{T} \boldsymbol{\Sigma}_{e}^{-1}\left(\mathbf{x}_{3}-2 \rho_{1} \mathbf{x}_{1}+\mathbf{x}_{2}\right), \\
& \boldsymbol{\Sigma}_{e}=\text { Toeplitz }\left([2+4 \rho_{1}^{2},-4 \rho_{1}, 1, \overbrace{0, \ldots, 0}^{N-5}]^{T}\right) .
\end{array}
$$

Now we make two relaxations. First, we relax the constraint of (30) as in (14). Second, as $\boldsymbol{\Sigma}_{e}$ is nonlinear in $\rho_{1}$ in (31), we substitute $\rho_{1}^{2}$ with a dummy variable $\beta$ and include a new relaxed constraint of $\beta \geq \rho_{1}^{2}$. To strengthen the constraint, we assume that the sign of $\rho_{1}$, denoted by $\Psi$, is known, which can be easily determined. As $\left|\rho_{1}\right| \leq 1$, we have $\Psi \rho_{1} \geq \rho_{1}^{2}$. As a result, a tighter constraint is $\Psi \rho_{1} \geq \beta \geq \rho_{1}^{2}$. As a result, the SDR formulation for approximating (27) is

$$
\begin{array}{ll}
\min _{v, \rho_{1}, \beta} & v \\
\text { s.t. } \quad & {\left[\begin{array}{cc}
v & \left(\mathbf{x}_{3}+\mathbf{x}_{2}-2 \rho_{1} \mathbf{x}_{1}\right)^{T} \\
\mathbf{x}_{3}+\mathbf{x}_{2}-2 \rho_{1} \mathbf{x}_{1} & \boldsymbol{\Sigma}_{e}
\end{array}\right] \geq \mathbf{0}_{N-1},} \\
& \boldsymbol{\Sigma}_{e}=\text { Toeplitz }\left([2+4 \beta,-4 \rho_{1}, 1, \overbrace{0, \ldots, 0}^{N-5}]^{T}\right), \\
& \Psi \rho_{1} \geq \beta \geq \rho_{1}^{2},
\end{array}
$$

where the matrix inverse operator that appears in (30) is removed by Schur complement representation. In our study, $\Psi$ is obtained using

$$
\Psi=\operatorname{sign}\left(\sum_{n=3}^{N} x(n-1)[x(n)+x(n-2)]\right) .
$$

After obtaining $\hat{\rho}_{1}$, the estimated frequency, $\hat{\omega}$, is computed as

$$
\widehat{\omega}=\cos ^{-1}\left(\hat{\rho}_{1}\right) .
$$

With the use of $\hat{\omega}$, the amplitude and phase are estimated according to constrained least squares:

$$
\begin{array}{ll}
\min _{\boldsymbol{\xi}} & (\mathbf{x}-\mathbf{B} \boldsymbol{\xi})^{T}(\mathbf{x}-\mathbf{B} \boldsymbol{\xi}) \\
\text { s.t. } & \boldsymbol{\xi}^{T} \boldsymbol{\xi}=A^{2},
\end{array}
$$

where

$$
\begin{gathered}
\mathbf{B}=\left[\begin{array}{cc}
\cos (\widehat{\omega}) & -\sin (\widehat{\omega}) \\
\cos (2 \widehat{\omega}) & -\sin (2 \hat{\omega}) \\
\vdots & \vdots \\
\cos (N \hat{\omega}) & -\sin (N \hat{\omega})
\end{array}\right], \\
\xi=\left[\begin{array}{l}
A \cos (\phi) \\
A \sin (\phi)
\end{array}\right],
\end{gathered}
$$

and $\mathbf{x}=[x(1), x(2), \ldots, x(N)]^{T}$. By expanding the objective function of (35) and removing $\mathbf{x}^{T} \mathbf{B}^{T} \mathbf{B x}$, and introducing a dummy matrix $\boldsymbol{\Xi}=\boldsymbol{\xi} \boldsymbol{\xi}^{T}$, (35) is equivalent to

$$
\begin{array}{ll}
\min _{\Xi, \xi} & \operatorname{tr}\left(\mathbf{B}^{T} \mathbf{B} \boldsymbol{\Xi}\right)-2 \mathbf{x}^{T} \mathbf{B} \boldsymbol{\xi} \\
\text { s.t. } & \operatorname{tr}(\boldsymbol{\Xi})=A^{2}, \\
& \boldsymbol{\Xi}=\boldsymbol{\xi} \boldsymbol{\xi}^{T} .
\end{array}
$$

Applying relaxation on $\Xi=\xi \xi^{T}$, the SDR estimator for $A$ and $\phi$ is

$$
\begin{array}{ll}
\min _{\boldsymbol{\Xi}, \boldsymbol{\xi}} & \operatorname{tr}\left(\mathbf{B}^{T} \mathbf{B} \boldsymbol{\Xi}\right)-2 \mathbf{x}^{T} \mathbf{B} \boldsymbol{\xi} \\
\text { s.t. } & \operatorname{tr}(\boldsymbol{\Xi})=A^{2}, \\
& {\left[\begin{array}{cc}
\boldsymbol{\Xi} & \boldsymbol{\xi} \\
\boldsymbol{\xi}^{T} & 1
\end{array}\right] \succeq \mathbf{0}_{3},}
\end{array}
$$

where we treat $A^{2}$ as a constant. Here, we have a convex program with unique optimum without dealing with multimodality as in the Lagrangian multiplier approach. Finally, the amplitude and phase estimates are computed as

$$
\begin{gathered}
\hat{A}=\sqrt{\operatorname{tr}(\boldsymbol{\Xi})}, \\
\hat{\phi}=\tan ^{-1}\left(\frac{[\xi]_{2}}{[\xi]_{1}}\right) .
\end{gathered}
$$


3.2. Nonlinear Least Squares Approach. Similar to Section 2.3, the NLS estimator for the real sinusoidal is

$$
\begin{aligned}
& \min _{\mathbf{s}, \omega, \phi, A}(\mathbf{x}-\mathbf{s})^{T} \boldsymbol{\Sigma}_{q}^{-1}(\mathbf{x}-\mathbf{s}) \\
& \text { s.t. } \quad s(n)=A \cos (\omega n+\phi), \quad n=1,2, \ldots, N,
\end{aligned}
$$

where $\mathbf{x}=[x(1), x(2), \ldots, x(N)]^{T}$ and $\mathbf{s}=[s(1), s(2)$, $\ldots, s(N)]^{T}$. Now we transform the constraint of $s(n)=$ $A \cos (\omega n+\phi)$ using the general LPP property as follows:

$$
\begin{aligned}
& s(n)+s(n-2 m) \\
& \quad=2 \rho_{m} s(n-m), \quad n=1,2, \cdots, N, m=1,2, \ldots, M,
\end{aligned}
$$

where $\rho_{m}=\cos (m \omega)$ and $M=\lfloor(N-1) / 2\rfloor$ is the maximum index for $m$. By considering various $n$ in (42), the set of equalities is converted to

$$
\begin{gathered}
\frac{s(i)+s(i-2 m)}{s(i-m)}=\frac{s(j)+s(j-2 m)}{s(j-m)}, \\
m=1,2, \ldots, M, \quad i>j=2 m+1,2 m+2, \ldots, N \\
\Longrightarrow s(i) s(j-m)+s(i-2 m) s(j-m) \\
=s(i-m) s(j)+s(i-m) s(j-2 m),
\end{gathered}
$$

where $\left\{\rho_{m}\right\}$ and $\omega$ are eliminated. Next, we define $\mathbf{S}=\mathbf{s s}^{T} \in \mathbb{R}^{N}$ with $[\mathbf{S}]_{i, j}=s(i) s(j)$ to write (43) as

$$
\begin{array}{r}
{[\mathbf{S}]_{i, j-m}+[\mathbf{S}]_{i-2 m, j-m}=[\mathbf{S}]_{i-m, j}+[\mathbf{S}]_{i-m, j-2 m},} \\
m=1,2, \ldots, M, \quad i>j=2 m+1,2 m+2, \ldots, N .
\end{array}
$$

Using similar procedure as in (20) and (21), the objective function of (41) is modified as $\operatorname{tr}\left(\boldsymbol{\Sigma}_{q}^{-1} \mathbf{S}\right)-2 \mathbf{s}^{T} \boldsymbol{\Sigma}_{q}^{-1} \mathbf{x}$. The $\mathrm{SDR}$ formulation is then given as

$$
\begin{array}{ll}
\min _{\mathbf{s}, \mathbf{S}} & \operatorname{tr}\left(\boldsymbol{\Sigma}_{q}^{-1} \mathbf{S}\right)-2 \mathbf{s}^{T} \boldsymbol{\Sigma}_{q}^{-1} \mathbf{x} \\
\text { s.t. } & {[\mathbf{S}]_{i, j-m}+[\mathbf{S}]_{i-2 m, j-m}=[\mathbf{S}]_{i-m, j}+[\mathbf{S}]_{i-m, j-2 m},} \\
& {\left[\begin{array}{cc}
\mathbf{S} & \mathbf{s} \\
\mathbf{s}^{T} & 1
\end{array}\right] \succeq \mathbf{0}_{N+1},} \\
& m=1,2, \ldots, M, \quad i>j=2 m+1,2 m+2, \ldots, N .
\end{array}
$$

As $\hat{\mathbf{s}}$ is the waveform estimate which is restricted to be a real tone, the parameter estimates are sequentially computed as

$$
\begin{gathered}
\hat{\omega}=\cos ^{-1}\left(\frac{\widehat{s}(1)+\widehat{s}(3)}{\widehat{s}(2)}\right), \\
\hat{\phi}=\tan ^{-1}\left(\frac{\widehat{s}(1) \cos (2 \widehat{\omega})-\widehat{s}(2) \cos (\widehat{\omega})}{\hat{s}(1) \sin (2 \widehat{\omega})-\widehat{s}(2) \sin (\widehat{\omega})}\right), \\
\hat{A}=\frac{\widehat{s}(1)}{\cos (\widehat{\omega}+\hat{\phi})} .
\end{gathered}
$$

\section{Multiple Complex Tones}

In this section, we generalize our development in Sections 2 and 3 to multiple complex sinusoids. Now the signal model is

$$
y(n)=s(n)+\eta(n), \quad n=1,2, \ldots, N,
$$

where

$$
s(n)=\sum_{m=1}^{L} A_{m} \exp \left\{j\left(\omega_{m} n+\phi_{m}\right)\right\}
$$

where $A_{m}>0, \omega_{m} \in(-\pi, \pi)$ and $\phi_{m} \in[0,2 \pi)$ represent the amplitude, frequency, and phase of $m$ th complex tone, respectively, and they are unknown constants with $\omega_{i} \neq \omega_{j}$, $i \neq j$, while $\eta$ is a zero-mean complex Gaussian noise. The number of sinusoids $L$ is assumed known. In the following, the SDR algorithm based on the LPP approach will be presented. Note that it is difficult to apply the NLS approach as there will be numerous cross-terms. Based on the linear prediction property for multiple complex tone signal, we have

$$
s(n)+\sum_{m=1}^{L} a_{m} s(n-m)=0,
$$

where

$$
\begin{aligned}
& a_{1}=-\sum_{i_{1}=1}^{L} \rho_{i_{1}}, \\
& a_{2}=\sum_{i_{1}=1}^{L} \sum_{i_{2}=1}^{i_{1}-1} \rho_{i_{1}} \rho_{i_{2}}, \\
& \quad \ldots \ldots \ldots \\
& a_{m}=(-1)^{m} \sum_{i_{1}=1}^{m} \sum_{i_{2}=1}^{i_{1}-1} \cdots \sum_{i_{m-1}=1}^{i_{m}-1} \prod_{j=1}^{m} \rho_{i_{j}}, \\
& \ldots \ldots \ldots \\
& a_{L}=(-1)^{L} \prod_{j=1}^{L} \rho_{L_{j}},
\end{aligned}
$$


and $\rho_{m}=\exp \left\{j \omega_{m}\right\}$. Note that $\left\{\rho_{m}\right\}$ are the roots of the polynomial with $\left\{a_{m}\right\}$ as coefficients. On the other hand, the LPP estimator for $\left\{a_{m}\right\}$ is

$$
\min _{\left\{a_{m}\right\}}\left[\mathbf{y}_{L+1}+\sum_{m=1}^{L} a_{m} \mathbf{y}_{m}\right]^{H} \boldsymbol{\Sigma}_{e}^{-1}\left[\mathbf{y}_{L+1}+\sum_{m=1}^{L} a_{m} \mathbf{y}_{m}\right],
$$

where $\mathbf{y}_{i}=[y(i), y(i+1), \ldots, y(N+L+1-i)]^{T}, i=$ $1,2, \ldots, L+1$ are the vectors of the observed signal, and $\boldsymbol{\Sigma}_{e}=E\left\{\mathbf{e e}^{H}\right\} \in \mathbb{C}^{N-L}$ with $\mathbf{e}=[e(1), e(2), \ldots, e(N-L)]^{T}$ and its elements are defined as

$$
\begin{aligned}
& e(n-L) \\
& \quad=y(n)+\sum_{m=1}^{L} a_{m} y(n-m), \quad n=L+1, L+2, \ldots, N .
\end{aligned}
$$

Assuming that $\boldsymbol{\Sigma}_{\eta}=\sigma_{\eta}^{2} \mathbf{I}_{N}, \boldsymbol{\Sigma}_{e}$ becomes

$$
\begin{aligned}
\boldsymbol{\Sigma}_{e}=\text { Toeplitz }( & {\left[\sum_{m=0}^{L} a_{m} a_{m}^{*}, \sum_{m=1}^{L} a_{m} a_{m-1}^{*}, \ldots,\right.} \\
& \sum_{m=\kappa}^{L} a_{m} a_{m-\kappa}^{*}, \ldots, a_{L} a_{0}^{*}, \overbrace{0, \ldots, 0}^{N-2 L-1}]) .
\end{aligned}
$$

As in the estimator for a single complex tone, we rewrite the optimization problem of (53) by including (55) as

$$
\begin{gathered}
\min _{\left\{a_{m}\right\}}\left[\mathbf{y}_{L+1}+\sum_{m=1}^{L} a_{m} \mathbf{y}_{m}\right]^{H} \mathbf{\Sigma}_{e}^{-1}\left[\mathbf{y}_{L+1}+\sum_{m=1}^{L} a_{m} \mathbf{y}_{m}\right] \\
\text { s.t. } \quad \boldsymbol{\Sigma}_{e}=\text { Toeplitz }\left(\left[\sum_{m=0}^{L} a_{m} a_{m}^{*}, \sum_{m=1}^{L} a_{m} a_{m-1}^{*}, \ldots,\right.\right. \\
\sum_{m=\kappa}^{L} a_{m} a_{m-\kappa}^{*}, \ldots, a_{L} a_{0}^{*}, \overbrace{0, \ldots, 0}^{N-2 L-1}]),
\end{gathered}
$$

which consists of cross-terms involving $\left\{a_{m}\right\}$ and resulting in a nonconvex program. We now define two dummy matrices $\mathbf{A} \in \mathbb{C}^{L+1}$ and $\mathbf{R} \in \mathbb{C}^{L+1}$ to replace $\left\{a_{m}\right\}$ and $\left\{\rho_{m}\right\}:$

$$
\begin{aligned}
& \mathbf{A}:=\left[\begin{array}{cc}
\mathbf{a} \mathbf{a}^{H} & \mathbf{a} \\
\mathbf{a}^{H} & 1
\end{array}\right], \\
& \mathbf{R}:=\left[\begin{array}{cc}
\boldsymbol{\rho} \boldsymbol{\rho}^{H} & \boldsymbol{\rho} \\
\boldsymbol{\rho}^{H} & 1
\end{array}\right],
\end{aligned}
$$

where $\boldsymbol{\rho}=\left[\rho_{1}, \rho_{2}, \ldots, \rho_{L}\right]^{T}$ and $\mathbf{a}=\left[a_{1}, a_{2}, \ldots, a_{L}\right]^{T}$. When A is introduced, the constraints will not be tight enough because the relation between entries of $\mathbf{A}$ and $\left\{a_{m}\right\}$ is relaxed. To compensate this, we look for relationships between the entries of $\mathbf{A}$ and $\mathbf{R}$, which is analogous to (43). For the $(L, L)$ entry of $\mathbf{A}$, we have

$$
[\mathbf{A}]_{L, L}=a_{L} a_{L}^{*}=\left|(-1)^{L} \prod_{i=1}^{L} \rho_{i}\right|^{2}=1 .
$$

For the $L$ th row and $L$ th column, the relations are

$$
\begin{aligned}
{[\mathbf{A}]_{m, L} } & =a_{m} a_{L}^{*} \\
& =(-1)^{L+m}\left[\sum_{i_{1}=1}^{m} \sum_{i_{2}=1}^{i_{1}-1} \ldots \sum_{i_{m-1}=1}^{i_{m}-1} \prod_{j=1}^{m} \rho_{i_{j}}\right]\left[\prod_{i=1}^{L} \rho_{i}\right]^{*} \\
& =(-1)^{L-m} \sum_{i_{1}=1}^{L-m} \sum_{i_{2}=1}^{i_{1}-1} \ldots \sum_{i_{L-m-1}}^{i_{L-m}-1} \prod_{j=1}^{L-m} \rho_{i_{j}} \\
& =a_{L-m}, \quad m=1,2, \ldots, L
\end{aligned}
$$

as $(-1)^{L+m}=(-1)^{L-m}$. Hence, $\mathbf{A}$ has the structure of

$$
\begin{aligned}
{[\mathbf{A}]_{m, k} } & =a_{m} a_{k}^{*}=a_{m} a_{k} a_{L}^{*} a_{L} \\
& =\left(a_{m} a_{L}^{*}\right)\left(a_{k} a_{L}^{*}\right)^{*}=a_{L-m} a_{L-k}^{*} \\
& =[\mathbf{A}]_{L-m, L-k}, \quad m, k=1,2, \ldots, L-1 .
\end{aligned}
$$

Finally, $[\mathbf{A}]_{1,1}$ and $\mathbf{R}$ are connected by

$$
[\mathbf{A}]_{1,1}=a_{1} a_{1}^{*}=\left|-\sum_{i_{1}=1}^{L} \rho_{i_{1}}\right|^{2}=\sum_{m=1}^{L} \sum_{k=1}^{L}[\mathbf{R}]_{m, k},
$$

and all the diagonal elements of $\mathbf{R}$ are

$$
[\operatorname{diag}(\mathbf{R}, 0)]_{m}=1, \quad m=1,2, \ldots, L .
$$

Constraints, which limit the degrees of freedom of $\mathbf{A}$ and $\mathbf{R}$ are obtained from (59)-(63). To fit (56) into convex framework, we replace $a_{i} a_{j}^{*}$ with $[\mathbf{A}]_{i, j}$. Furthermore, by introducing a dummy variable $v$, we make the objective function of (53) to a constraint. As a result, the optimization problem is now

$$
\begin{array}{rl}
\min _{\mathbf{A},\left\{a_{m}\right\}, v} & v \\
\text { s.t. } \quad v= & {\left[\mathbf{y}_{L+1}+\sum_{m=1}^{L} a_{m} \mathbf{y}_{m}\right]^{H} \boldsymbol{\Sigma}_{e}^{-1}\left[\mathbf{y}_{L+1}+\sum_{m=1}^{L} a_{m} \mathbf{y}_{m}\right],} \\
\boldsymbol{\Sigma}_{e}= & \operatorname{Toeplitz}\left(\left[\sum_{m=0}^{L}[\mathbf{A}]_{m, m}, \sum_{m=1}^{L}[\mathbf{A}]_{m, m-1}, \ldots,\right.\right. \\
\sum_{m=\kappa}^{L}[\mathbf{A}]_{m, m-\kappa}, \ldots,[\mathbf{A}]_{L, 0}, \overbrace{0, \ldots, 0,}^{N-2 L-1}]), & \rho_{1}+\rho_{2}+\cdots+\rho_{L},
\end{array}
$$

where the last constraint comes from the definition of $a_{1}$ and we have defined $[\mathbf{A}]_{0,0}=1,[\mathbf{A}]_{m, 0}=a_{m}$ and $[\mathbf{A}]_{0, m}=a_{m}^{*}$, 
$m=1,2 \ldots, L$. Using Schur complement representation and performing SDR relaxation, (64) becomes

$$
\begin{aligned}
& \min _{\mathbf{R}, \mathbf{A},\left\{a_{m}\right\},\left\{\rho_{m}\right\}, v} v \\
& \text { s.t. } \left.\left.\left[\begin{array}{c}
v \\
\mathbf{y}_{L+1}+\sum_{m=1}^{L} a_{m} \mathbf{y}_{m}
\end{array}\right] \mathbf{y}_{L+1}+\sum_{m=1}^{L} a_{m} \mathbf{y}_{m}\right]^{H}\right] \mathbf{0}_{N-\mathrm{L}+1} \text {, } \\
& \boldsymbol{\Sigma}_{e}=\text { Toeplitz }\left(\left[\sum_{m=0}^{L}[\mathbf{A}]_{m, m}, \sum_{m=1}^{L}[\mathbf{A}]_{m, m-1}, \ldots,\right.\right. \\
& \sum_{m=\kappa}^{L}[\mathbf{A}]_{m, m-\kappa}, \ldots,[\mathbf{A}]_{L, 0}, \overbrace{0, \ldots, 0}^{N-2 L-1}]), \\
& \mathbf{R} \succeq 0_{L+1}, \\
& \mathbf{A} \succeq 0_{L+1}, \\
& a_{1}=\rho_{1}+\rho_{2}+\cdots+\rho_{L}, \\
& {[\mathbf{A}]_{m, k}=[\mathbf{A}]_{L-m, L-k}, \quad m, k=1,2, \ldots, L,} \\
& {[\mathbf{A}]_{1,1}=\sum_{m=1}^{L} \sum_{k=1}^{L}[\mathbf{R}]_{m, k},} \\
& {[\mathbf{A}]_{L, L}=1 \text {, }} \\
& {[\operatorname{diag}(\mathbf{R}, 0)]_{m}=1, \quad m=1,2, \ldots, L,}
\end{aligned}
$$

where the last four constraints are based on (59)-(63) to limit the matrix structure. It is worthy to mention that although constraints are imposed, some entries are not well bounded which may decrease the estimation accuracy. Note also that similar to (32), the inverse matrix operator is removed from the Schur complement representation. The frequencies are estimated by finding the phases of $\left\{\hat{\rho}_{m}\right\}$. After obtaining $\left\{\widehat{\omega}_{m}\right\},\left\{\widehat{A}_{m}\right\}$ and $\left\{\hat{\phi}_{m}\right\}$ are computed similarly as in (35):

$$
\begin{aligned}
& \min _{\xi,\left\{A_{m}^{2}\right\}}(\mathbf{x}-\mathbf{B} \boldsymbol{\xi})^{H}(\mathbf{x}-\mathbf{B} \boldsymbol{\xi}) \\
& \text { s.t. }\left[\operatorname{diag}\left(\xi \xi^{H}, 0\right)\right]_{m}=A_{m}^{2}, \quad m=1,2, \ldots, L,
\end{aligned}
$$

where

$$
\begin{gathered}
\mathbf{B}=\left[\begin{array}{cccc}
\exp \left\{j \hat{\omega}_{1}\right\} & \exp \left\{j \hat{\omega}_{2}\right\} & \cdots & \exp \left\{j \hat{\omega}_{L}\right\} \\
\exp \left\{j 2 \hat{\omega}_{1}\right\} & \exp \left\{j 2 \hat{\omega}_{2}\right\} & \cdots & \exp \left\{j 2 \hat{\omega}_{L}\right\} \\
\vdots & \vdots & \vdots & \vdots \\
\exp \left\{j N \hat{\omega}_{1}\right\} & \exp \left\{j N \hat{\omega}_{2}\right\} & \cdots & \exp \left\{j N \hat{\omega}_{L}\right\}
\end{array}\right], \\
\boldsymbol{\xi}=\left[A_{1} \exp \left\{j \phi_{1}\right\}, A_{2} \exp \left\{j \phi_{2}\right\}, \ldots, A_{L} \exp \left\{j \phi_{L}\right\}\right]^{T} .
\end{gathered}
$$

By expanding (66) and dropping the irrelevant terms, (66) is equivalent to

$$
\begin{aligned}
& \min _{\xi,\left\{A_{m}^{2}\right\}} \boldsymbol{\xi}^{H} \mathbf{B}^{H} \mathbf{B} \boldsymbol{\xi}-2 \mathbf{y}^{H} \mathbf{B} \boldsymbol{\xi} \\
& \text { s.t. }\left[\operatorname{diag}\left(\boldsymbol{\xi} \xi^{H}, 0\right)\right]_{m}=A_{m}^{2}, \quad m=1,2, \ldots, L .
\end{aligned}
$$

We then define a matrix $\boldsymbol{\Xi}=\boldsymbol{\xi} \boldsymbol{\xi}^{H}$ and perform relaxation on (68) to yield

$$
\begin{array}{ll}
\min _{\boldsymbol{\Xi}, \boldsymbol{\xi},\left\{A_{m}^{2}\right\}} \operatorname{tr}\left(\mathbf{B}^{H} \mathbf{B} \boldsymbol{\Xi}\right)-2 \mathbf{y}^{H} \mathbf{B} \boldsymbol{\xi} \\
\text { s.t. } \quad[\operatorname{diag}(\boldsymbol{\Xi}, 0)]_{m}=A_{m}^{2}, \quad m=1,2, \ldots, L, \\
& {\left[\begin{array}{cc}
\boldsymbol{\Xi} & \boldsymbol{\xi} \\
\boldsymbol{\xi}^{H} & 1
\end{array}\right] \succeq \mathbf{0}_{L+1},}
\end{array}
$$

where $\left\{A_{m}^{2}\right\}$ are treated as constants. The amplitude and phase estimates are computed as

$$
\begin{gathered}
\hat{A}_{m}=\sqrt{[\operatorname{diag}(\boldsymbol{\Xi}, 0)]_{m}}, \quad m=1,2, \ldots, L, \\
\hat{\phi}_{m}=\angle\left([\boldsymbol{\xi}]_{m}\right), \quad m=1,2, \ldots, L .
\end{gathered}
$$

As an illustration, we consider $L=2$ as follows. The linear prediction property of (51) gives

$$
s(n)+a_{1} s(n-1)+a_{2} s(n-2)=0,
$$

where $a_{1}=\rho_{1}+\rho_{2}$ and $a_{2}=\rho_{1} \rho_{2}$. The covariance matrix is

$$
\begin{aligned}
& \Sigma_{e} \\
& =\text { Toeplitz }\left(\left[1+a_{1} a_{2}^{*}+a_{2} a_{2}^{*}, a_{1}+\right.\right. \\
& a_{1} a_{2}^{*}, a_{2}^{*}, \overbrace{0, \ldots, 0}^{N-5}]) \in \mathbb{C}^{N-2} .
\end{aligned}
$$

From (59) and (62), and replacing $a_{i} a_{i}^{*}$ with $[\mathbf{A}]_{i, i}, i=1,2$, we have

$$
\begin{aligned}
1+a_{1} a_{1}^{*}+a_{2} a_{2}^{*} & =1+[\mathbf{A}]_{1,1}+[\mathbf{A}]_{2,2} \\
& =1+\sum_{m=1}^{2} \sum_{k=1}^{2}[\mathbf{R}]_{m, k}+1 .
\end{aligned}
$$

From (61), we get

$$
\begin{aligned}
a_{1}+a_{2} a_{1}^{*} & =a_{1}+[\mathbf{A}]_{2,1} \\
& =a_{1}+[\mathbf{A}]_{2-2,2-1}=a_{1}+a_{1}^{*}
\end{aligned}
$$


Applying (74) and (75) yields

$$
\begin{aligned}
& \min _{a_{1}, a_{2}, \rho_{1}, \rho_{2}, v} v \\
& \text { s.t. } \quad v=\left(\mathbf{y}_{3}+a_{1} \mathbf{y}_{1}+a_{2} \mathbf{y}_{2}\right)^{H} \boldsymbol{\Sigma}_{e}^{-1}\left(\mathbf{y}_{3}+a_{1} \mathbf{y}_{1}+a_{2} \mathbf{y}_{2}\right), \\
& a_{1}=\rho_{1}+\rho_{2}, \\
& \mathbf{\Sigma}_{e}=\text { Toeplitz }\left(\left[2+\sum_{m=1}^{2} \sum_{k=1}^{2}[\mathbf{R}]_{m, k}, a_{1}+\right.\right. \\
& a_{1}^{*}, a_{2}^{*}, \overbrace{0, \ldots, 0}^{N-5}]) .
\end{aligned}
$$

Finally, the SDR algorithm for frequencies is

$$
\begin{aligned}
& \min _{\mathbf{R}, \mathbf{A}, a_{1}, a_{2}, \rho_{1}, \rho_{2}, v} v \\
& \text { s.t. }\left[\begin{array}{cc}
v & \left(\mathbf{x}_{3}+a_{1} \mathbf{x}_{1}+a_{2} \mathbf{x}_{2}\right)^{H} \\
\mathbf{x}_{3}+a_{1} \mathbf{x}_{1}+a_{2} \mathbf{x}_{2} & \boldsymbol{\Sigma}_{e}
\end{array}\right] \succeq \mathbf{0}_{N-1} \text {, } \\
& \mathrm{A} \succeq \mathbf{0}_{3}, \\
& \mathbf{R} \succeq \mathbf{0}_{3}, \\
& \boldsymbol{\Sigma}_{e}=\text { Toeplitz }\left(\left[2+\sum_{m=1}^{2} \sum_{k=1}^{2}[\mathbf{R}]_{m, k}, a_{1}+\right.\right. \\
& a_{1}^{*}, a_{2}^{*}, \overbrace{0, \ldots, 0}^{N-5}]) \\
& a_{1}=\rho_{1}+\rho_{2}, \\
& {[\operatorname{diag}(\mathbf{R}, 0)]_{m}=1, \quad m=1,2 .}
\end{aligned}
$$

Using (69)-(71), $\hat{A}_{1}, \hat{\phi}_{1}, \hat{A}_{2}$, and $\hat{\phi}_{2}$ can then be determined. For $L=2, \mathbf{B}$ and $\xi$ have the forms of

$$
\begin{gathered}
\mathbf{B}=\left[\begin{array}{cc}
\exp \left\{j \hat{\omega}_{1}\right) & \exp \left\{j \hat{\omega}_{2}\right\} \\
\exp \left\{j 2 \widehat{\omega}_{1}\right) & \exp \left\{j 2 \widehat{\omega}_{2}\right\} \\
\vdots & \vdots \\
\exp \left\{j N \hat{\omega}_{1}\right) & \exp \left\{j N \hat{\omega}_{2}\right\}
\end{array}\right], \\
\boldsymbol{\xi}=\left[A_{1} \exp \left\{j \phi_{1}\right\}, A_{2} \exp \left\{j \phi_{2}\right\}\right]^{T} .
\end{gathered}
$$

It is noteworthy that the real signal model can be tackled in a similar manner by including the two additional constraints: $\left\{a_{m}\right\}$ are symmetric and $\left\{\rho_{m}\right\}$ are in conjugate pairs.

\section{Two-Dimensional Complex Tone}

In this section, we will extend the periodogram and NLS approaches in Section 2 to parameter estimation of a $2 \mathrm{D}$ complex sinusoid in additive noise. It is straightforward to

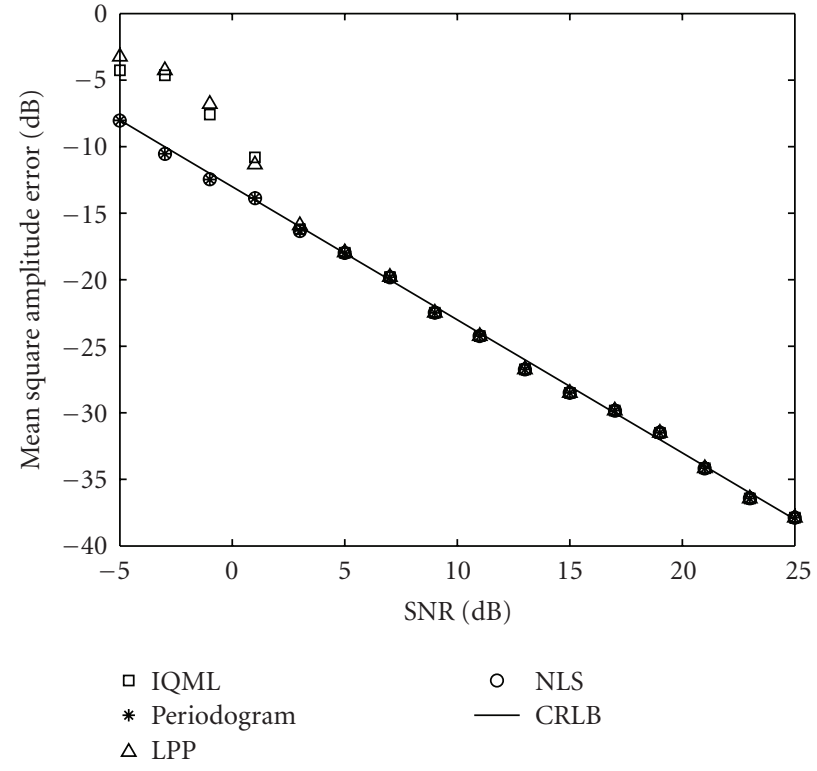

Figure 1: MSE for $A$ of single complex sinusoid at $N=20$.

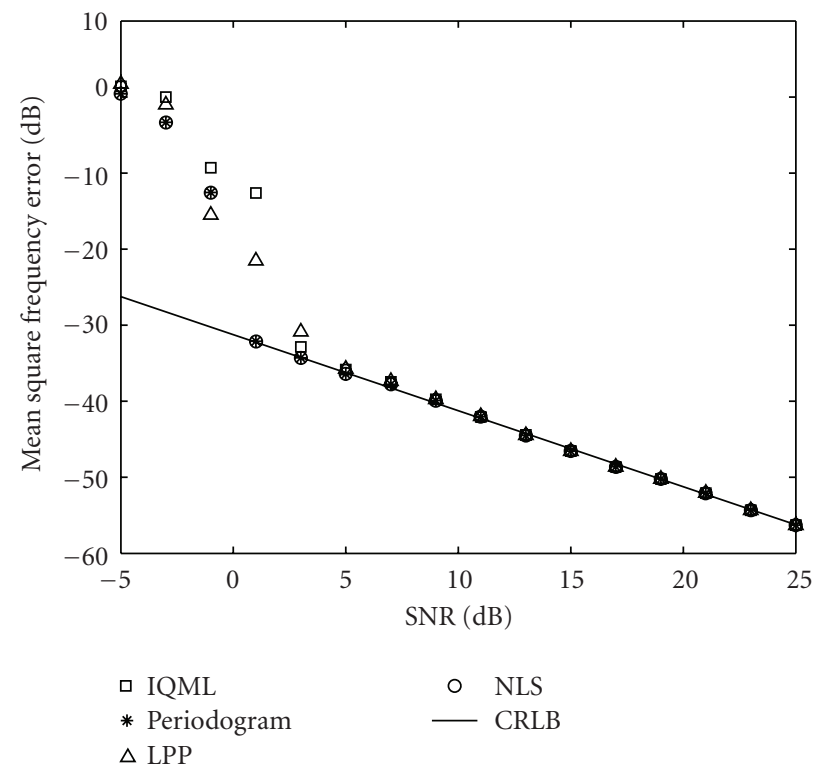

FIGURE 2: MSE for $\omega$ of single complex sinusoid at $N=20$.

apply the LPP approach although its formulation is more tedious. The observed 2D signal is modeled as

$$
\begin{aligned}
y(m, n)= & A \exp \{j(\mu m+\nu n+\phi)\} \\
& +\eta(m, n), \quad m=1,2, \ldots, M, n=1,2, \ldots, N,
\end{aligned}
$$

where $A$ is the unknown amplitude, $\mu$ and $\nu$ are the unknown $2 \mathrm{D}$ frequencies, and $\phi$ is the initial phase while $\eta(m, n)$ is a zero-mean Gaussian noise. The task is to find $\mu, v, A, \phi$ from the $M N$ samples of $\{y(m, n)\}$. To facilitate the algorithm 

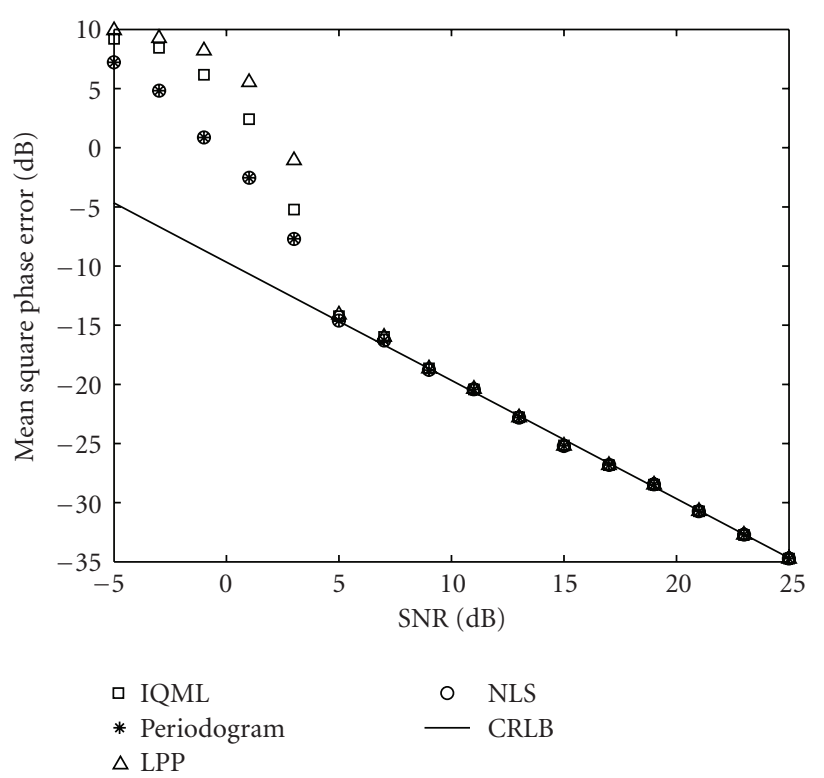

FIgUre 3: MSE for $\phi$ of single complex sinusoid at $N=20$.

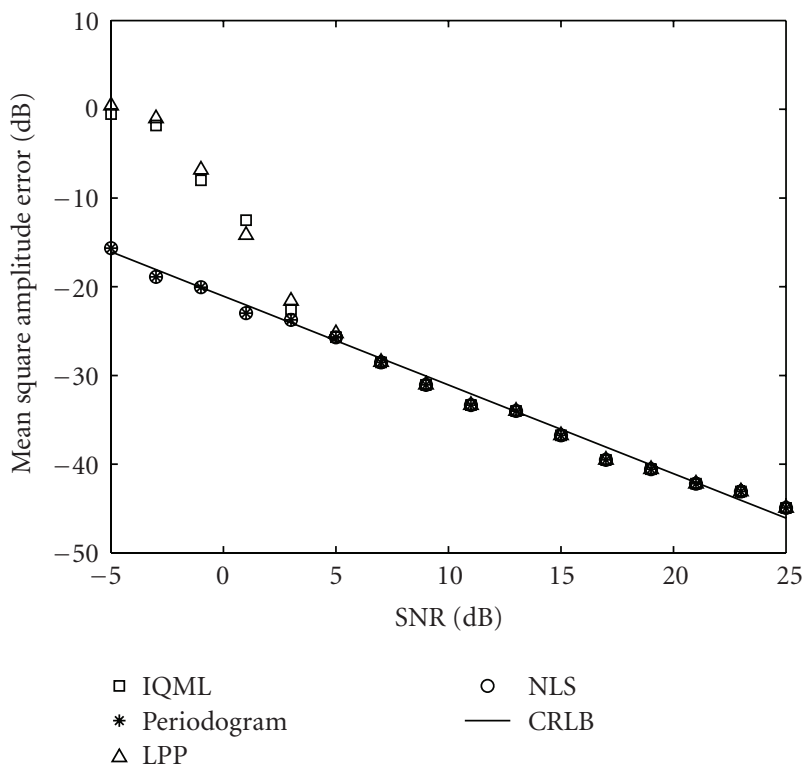

Figure 4: MSE for $A$ of single complex sinusoid at $N=128$.

development, we let $[\mathbf{S}]_{m, n}=A \exp \{j(\mu m+\nu n+\phi)\},[\mathbf{Y}]_{m, n}=$ $y(m, n)$ and $[\mathbf{N}]_{m, n}=\eta(m, n)$ to rewrite $(79)$ as

$$
\mathbf{Y}=\mathbf{S}+\mathbf{N} \text {. }
$$

5.1. Periodogram Approach. Assuming that $\{\eta(m, n)\}$ are IID or $E\left\{\operatorname{vec}(\mathbf{N})^{H} \operatorname{vec}(\mathbf{N})\right\}=\sigma_{\eta}^{2} \mathbf{I}_{M N}$ with unknown $\sigma_{\eta}^{2}$, the ML frequency estimates can be obtained from

$$
\max _{\mu, \nu}|y(\mu, \nu)|^{2},
$$
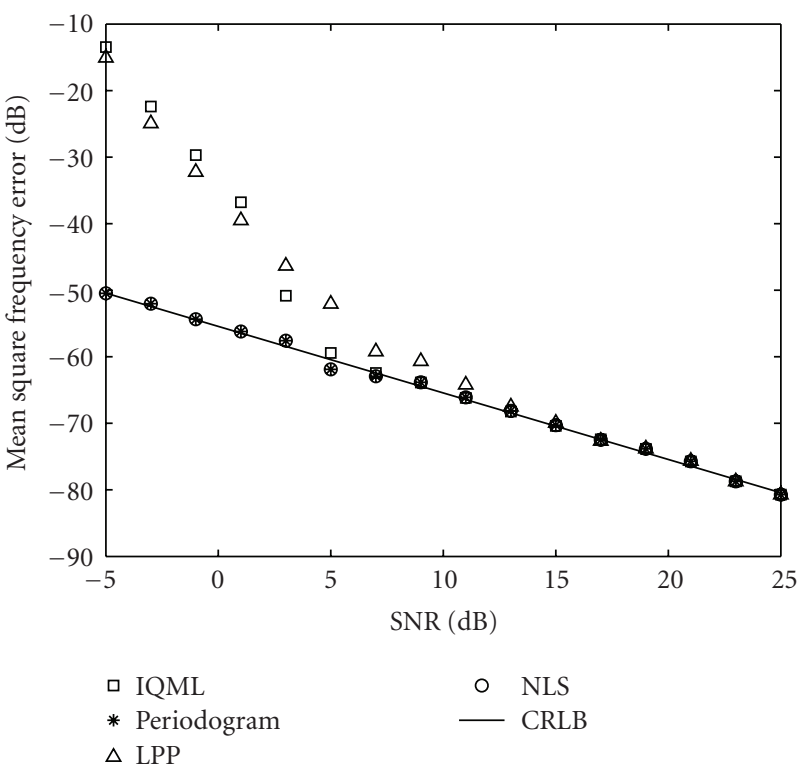

Figure 5: MSE for $\omega$ of single complex sinusoid at $N=128$.

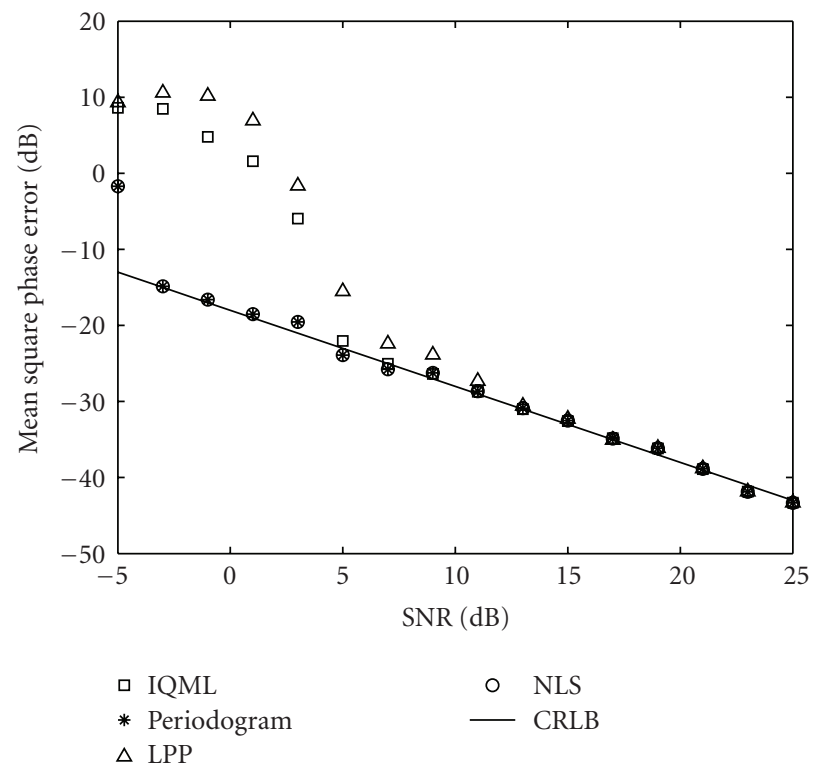

Figure 6: MSE for $\phi$ of single complex sinusoid at $N=128$.

where $\mathcal{y}(\mu, \nu)=\sum_{m=1}^{M} \sum_{n=1}^{N} y(m, n) \exp \{-j(\mu m+\nu n)\}$ is the 2D DTFT of $y(m, n)$. By introducing a dummy matrix $\mathrm{C}$ which is analogous to $\mathrm{c}$ in the single complex tone model, the optimization problem of (81) is equivalent to

$$
\begin{aligned}
& \max _{\mathbf{C}, \mu, \nu}\left|\operatorname{vec}(\mathbf{C})^{H} \operatorname{vec}(\mathbf{Y})\right|^{2} \\
& \text { s.t. } \quad[\mathbf{C}]_{m, n}=\exp \{j(\mu m+\nu n)\} .
\end{aligned}
$$




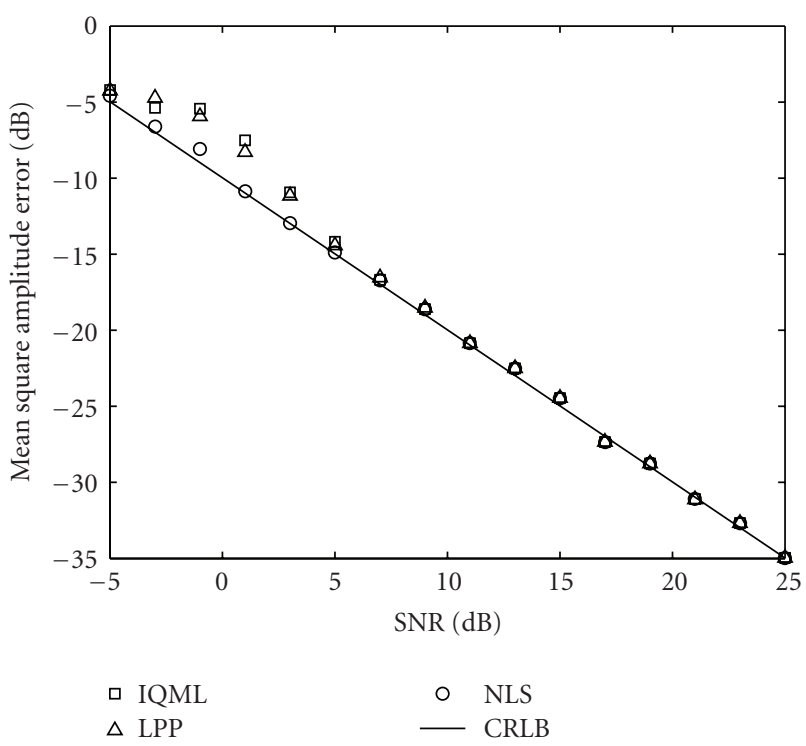

Figure 7: MSE for $A$ of single real sinusoid with fixed $\phi$.

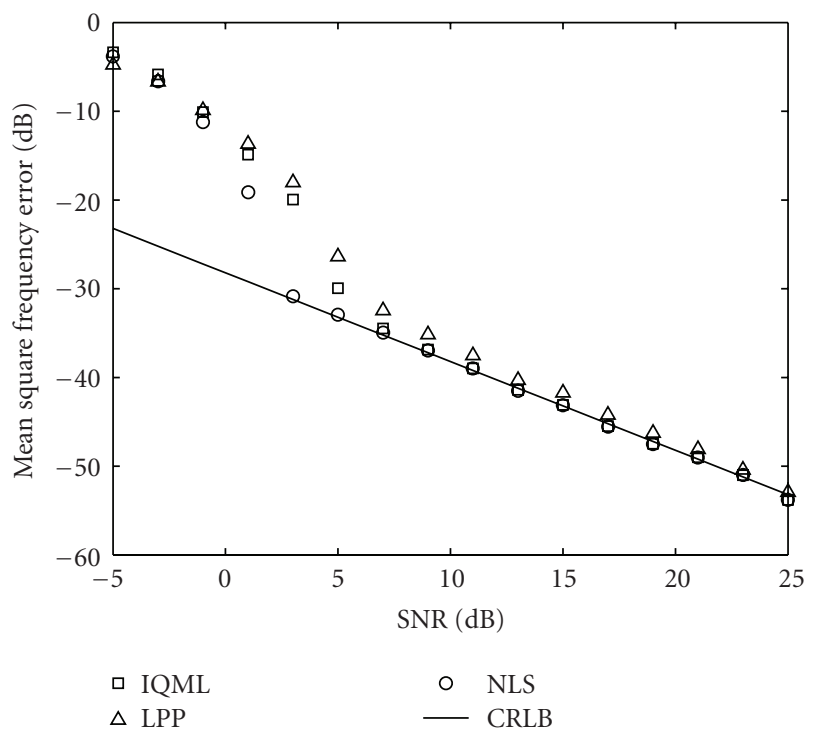

Figure 8: MSE for $\omega$ of single real sinusoid with fixed $\phi$.
Applying SDR on (83) and retaining the Toeplitz structure of $C$ yields the estimator for $2 \mathrm{D}$ frequencies:

$$
\begin{aligned}
& \max _{\mathbf{C}, \mathbf{C}, \mu, \nu} \quad \operatorname{vec}(\mathbf{Y})^{H} \mathbf{C} \operatorname{vec}(\mathbf{Y}) \\
& \text { s.t. } \quad\left[\begin{array}{cc}
\mathbf{C} & \operatorname{vec}(\mathbf{C}) \\
\operatorname{vec}(\mathbf{C})^{H} & 1
\end{array}\right] \succeq \mathbf{0}_{(M N+1) \times(M N+1)}, \\
& \mathbf{C}=\operatorname{blkdiag}(\overbrace{\mathbf{C}^{(\mu)}, \mathbf{C}^{(\mu)}, \ldots, \mathbf{C}^{(\mu)}}^{N}), \\
& \left.\operatorname{perm}_{1}(\mathbf{C}, M, N)=\operatorname{blkdiag}^{\left(\nu \mathbf{C}^{(\nu)}, \mathbf{C}^{(\nu)}, \ldots, \mathbf{C}^{(\nu)}\right.}\right), \\
& \operatorname{perm}_{2}(\mathbf{C}, M, N)=\mathbf{C}^{(\mu+\nu)}, \\
& \mathbf{C}^{(\kappa)}=\operatorname{Toeplitz}\left(\left[c_{0}^{(\kappa)}, c_{1}^{(\kappa) *}, \ldots, c_{N-1}^{(\kappa) *}\right]\right), \\
& \kappa=\mu, \nu, \mu+\nu .
\end{aligned}
$$

Due to the Toeplitz structure, $c_{n}^{(\kappa)}$ has the form of $\exp \{j \kappa n\}$. The $\mathbf{C}$ and $\operatorname{perm}_{1}(\mathbf{C}, M, N)$ are used to maintain the Toeplitz structure in vertical and horizontal directions, respectively, and the ideal expression of the latter is $\operatorname{vec}\left(\mathbf{C}^{T}\right)^{H} \operatorname{vec}\left(\mathbf{C}^{T}\right)$. For the entries of $\mathbf{C}$ depicting the form of $\exp \{j(\mu+\nu) n\}$, $n=1,2, \ldots, \min (M, N)$, where $(\mu+\nu)$ is considered as a frequency parameter, perm ${ }_{2}(\mathbf{C}, M, N)$ is employed to impose the corresponding Toeplitz relation. (See the appendix for the perm function development.) The frequency estimates are computed as

$$
\hat{\mu}=\angle\left(\left[\mathbf{C}^{(\mu)}\right]_{2,1}\right), \quad \hat{\nu}=\angle\left(\left[\mathbf{C}^{(\nu)}\right]_{2,1}\right) .
$$

With the use of the estimated $\mathbf{C}$, the LS amplitude and phase estimates, denoted by $\hat{A}$ and $\hat{\phi}$, are

$$
\begin{gathered}
\hat{A}=\frac{\left|\operatorname{tr}\left(\mathbf{Y} \mathbf{C}^{H}\right)\right|}{M N}, \\
\hat{\phi}=\angle\left(\operatorname{tr}\left(\mathbf{Y} \mathbf{C}^{H}\right)\right) .
\end{gathered}
$$

We further introduce a dummy matrix $\mathbf{C}=$ $\operatorname{vec}(\mathbf{C}) \operatorname{vec}(\mathbf{C})^{H} \in \mathbb{C}^{M N}$ to replace $\left|\operatorname{vec}(\mathbf{C})^{H} \operatorname{vec}(\mathbf{Y})\right|^{2}$ with vec $(\mathbf{Y})^{H} \mathbf{C} \operatorname{vec}(\mathbf{Y})$ to rewrite (82) as

$$
\begin{array}{ll}
\max _{\mathbf{C}, \mathbf{C}, \mu, \nu} & \operatorname{vec}(\mathbf{Y})^{H} \mathbf{C} \operatorname{vec}(\mathbf{Y}) \\
\text { s.t. } \quad & \mathbf{C}=\operatorname{vec}(\mathbf{C}) \operatorname{vec}(\mathbf{C})^{H}, \\
& {[\mathbf{C}]_{m, n}=\exp \{j(\mu m+\nu n)\} .}
\end{array}
$$

5.2. Nonlinear Least Squares Approach. Denoting the noise covariance matrix as $\boldsymbol{\Sigma}_{N}=\mathrm{E}\left\{\operatorname{vec}(\mathbf{N}) \operatorname{vec}(\mathbf{N})^{H}\right\}$, the NLS estimator for $2 \mathrm{D}$ sinusoidal parameters is

$$
\begin{gathered}
\min _{\mathbf{S}, A, \mu, \nu, \phi}(\operatorname{vec}(\mathbf{Y})-\operatorname{vec}(\mathbf{S}))^{H} \mathbf{\Sigma}_{N}^{-1}(\operatorname{vec}(\mathbf{Y})-\operatorname{vec}(\mathbf{S})) \\
\text { s.t. } \quad[\mathbf{S}]_{m, n}=A \exp \{j(\mu m+\nu n+\phi)\} \\
m, n=1,2, \ldots, M .
\end{gathered}
$$




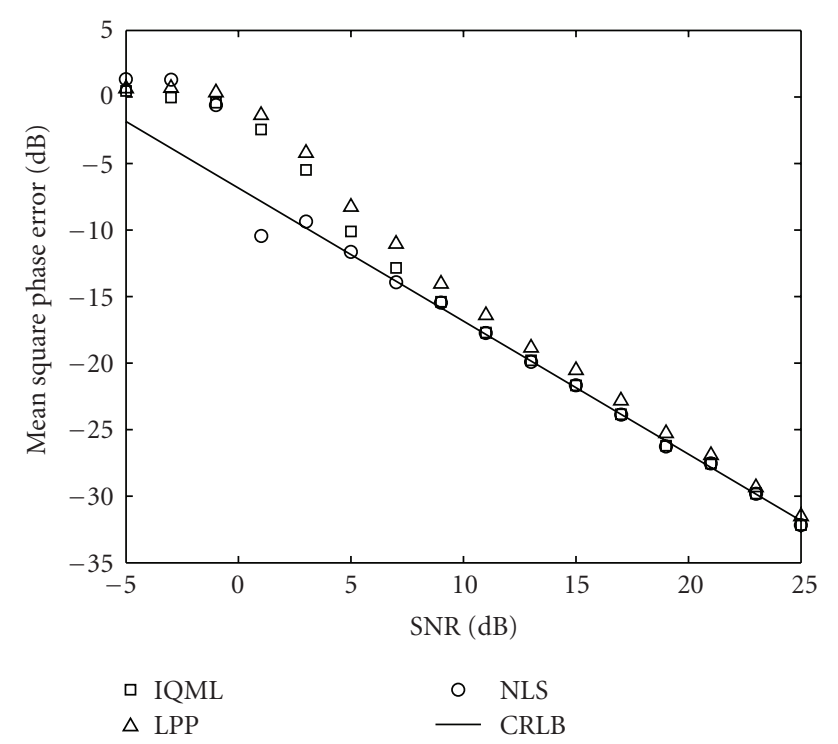

FIGURE 9: MSE for $\phi$ of single real sinusoid with fixed $\phi$.

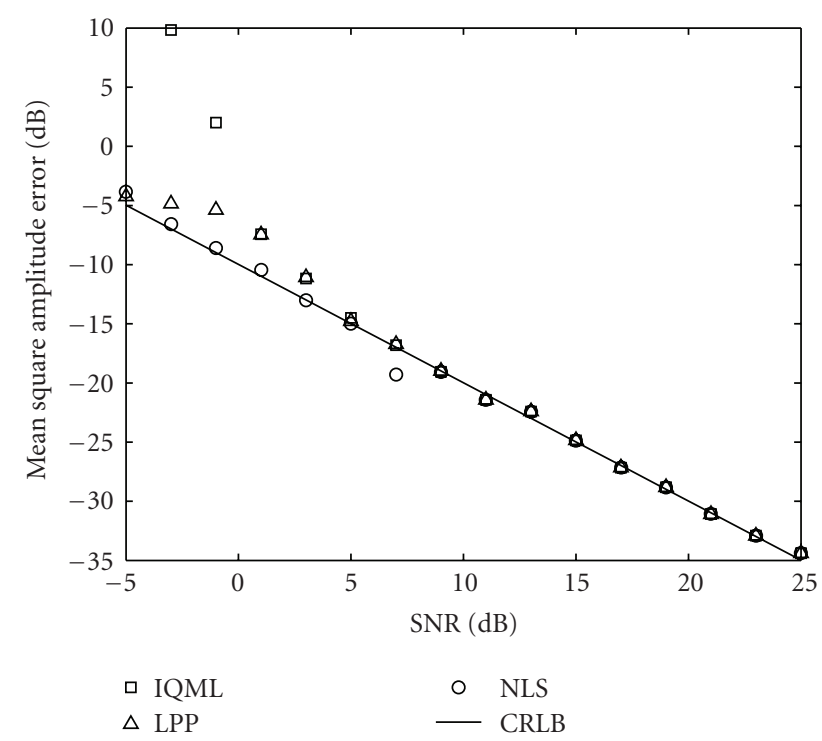

FIGURE 10: MSE for $A$ of single real sinusoid with random $\phi$.

Introducing a dummy matrix $\mathbf{S}=\operatorname{vec}(\mathbf{S}) \operatorname{vec}(\mathbf{S})^{H},(88)$ is equivalent to

$$
\begin{array}{cl}
\min _{\mathbf{S}, \mathbf{S}} & \operatorname{tr}\left(\boldsymbol{\Sigma}^{-1} \mathbf{S}\right)-\operatorname{vec}(\mathbf{S})^{H} \boldsymbol{\Sigma}_{N}^{-1} \operatorname{vec}(\mathrm{Y})-\operatorname{vec}(\mathbf{Y})^{H} \boldsymbol{\Sigma}^{-1} \operatorname{vec}(\mathbf{S}) \\
\text { s.t. } & \mathbf{S}=\operatorname{vec}(\mathbf{S}) \operatorname{vec}(\mathbf{S})^{H} \\
& {[\mathbf{S}]_{m, n}=A \exp \{j(\mu m+\nu n+\phi)\}} \\
m, n=1,2, \cdots, M .
\end{array}
$$

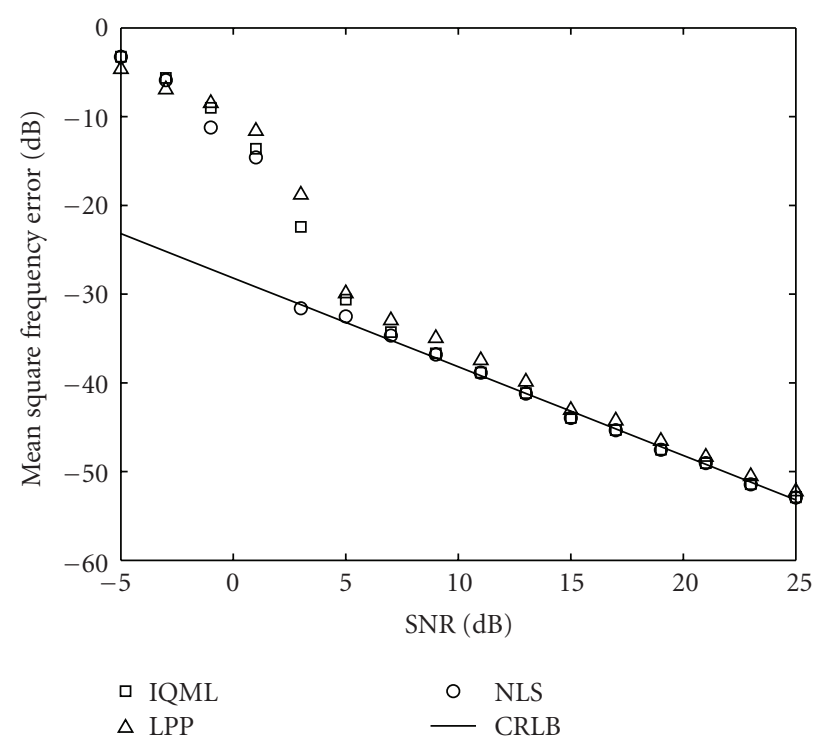

FIGURE 11: MSE for $\omega$ of single real sinusoid with random $\phi$.

Performing relaxation and maintaining Toeplitz structure on $S$ yields the SDR formulation:

$$
\begin{aligned}
& \min _{\mathbf{S}, \mathbf{S}} \operatorname{tr}\left(\boldsymbol{\Sigma}^{-1} \mathbf{S}\right)-\operatorname{vec}(\mathbf{S})^{H} \boldsymbol{\Sigma}_{N}^{-1} \operatorname{vec}(\mathbf{Y})-\operatorname{vec}(\mathbf{Y})^{H} \boldsymbol{\Sigma}^{-1} \operatorname{vec}(\mathbf{S}) \\
& \text { s.t. } \quad\left[\begin{array}{cr}
\mathbf{S} & \operatorname{vec}(\mathbf{S}) \\
\operatorname{vec}(\mathbf{S})^{H} & 1
\end{array}\right] \geq \mathbf{0}_{N M+1 \times M N+1}, \\
& \mathbf{S}=\operatorname{blkdiag}(\overbrace{\mathbf{S}^{(\mu)}, \mathbf{S}^{(\mu)}, \ldots, \mathbf{S}^{(\mu)}}^{N}), \\
& \operatorname{perm}_{1}(\mathbf{S}, M, N)=\operatorname{blkdiag}(\overbrace{\mathbf{S}^{(\nu)}, \mathbf{S}^{(\nu)}, \ldots, \mathbf{S}^{(\nu)}}^{M}), \\
& \operatorname{perm}_{2}(\mathbf{S}, M, N)=\mathbf{S}^{(\mu+\nu)}, \\
& \mathbf{S}^{(\kappa)}=\operatorname{Toeplitz}\left(\left[c_{0}^{(\kappa)}, c_{1}^{(\kappa) *}, \ldots, c_{N-1}^{(\kappa) *}\right]\right), \quad \kappa=\mu, \nu, \mu+\nu,
\end{aligned}
$$

where $c_{n}^{(\kappa)}$ will be of the form of $A^{2} \exp \{j \kappa n\}$. The $\operatorname{perm}_{1}(\mathbf{S}, M, N)$ and $\operatorname{perm}_{2}(\mathbf{S}, M, N)$ perform similar functions as in the periodogram approach but with a scaling of $A^{2}$. From the estimate of $c_{1}^{(\kappa)}$, denoted by $\widehat{c}_{1}^{(\kappa)}, \kappa=\mu, \nu$, the $2 \mathrm{D}$ frequency estimates are calculated as

$$
\hat{\mu}=\angle\left(\widehat{c}_{1}^{(\mu)}\right), \quad \hat{\nu}=\angle\left(\hat{c}_{1}^{(\nu)}\right),
$$

while the amplitude and phase estimates are computed as

$$
\begin{gathered}
\hat{A}=\sqrt{[\mathbf{S}]_{1,1}}, \\
\hat{\phi}=\angle\left([\mathbf{S}]_{1,1} c_{1}^{(\mu+\nu) *}\right) .
\end{gathered}
$$




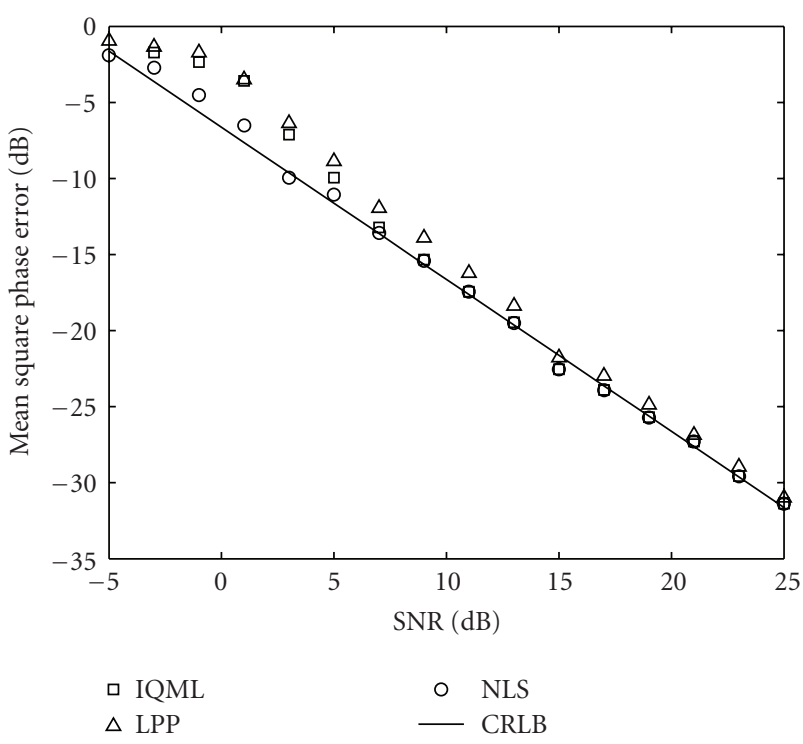

FIGURE 12: MSE for $\phi$ of single real sinusoid with random $\phi$.

In particular, when the noises are uncorrelated among rows or columns, we can break down the huge SDR constraint of $\mathbf{S}$ and (90) is simplified as

$$
\begin{aligned}
& \min _{\mathbf{S}, \mathbf{S}} \operatorname{tr}\left(\boldsymbol{\Sigma}_{N}^{-1} \mathbf{S}\right)-\operatorname{vec}(\mathbf{S})^{H} \boldsymbol{\Sigma}_{N}^{-1} \operatorname{vec}(\mathbf{Y})-\operatorname{vec}(\mathbf{Y})^{H} \boldsymbol{\Sigma}_{N}^{-1} \operatorname{vec}(\mathbf{S}) \\
& \text { s.t. }\left[\begin{array}{cc}
\mathbf{S}^{(\mu)} & \mathbf{s}_{n}^{(\mu)} \\
\mathbf{s}_{n}^{(\mu) H} & 1
\end{array}\right] \succeq \mathbf{0}_{M+1}, \quad n=1,2, \ldots, N, \\
& {\left[\begin{array}{cc}
\mathbf{S}^{(v)} & \mathbf{s}_{m}^{(\nu)} \\
\mathbf{s}_{m}^{(v) H} & 1
\end{array}\right] \succeq \mathbf{0}_{N+1}, \quad m=1,2, \ldots, M,} \\
& {\left[\begin{array}{cc}
\mathbf{S}^{(\mu+\nu)} & \operatorname{diag}(\mathbf{S}, 0) \\
\operatorname{diag}(\mathbf{S}, 0)^{H} & 1
\end{array}\right] \succeq \mathbf{0}_{\min (M, N)+1},} \\
& \mathbf{S}=\operatorname{blkdiag}(\overbrace{\mathbf{S}^{(\mu)}, \mathbf{S}^{(\mu)}, \ldots, \mathbf{S}^{(\mu)}}^{N}) \text {, } \\
& \operatorname{perm}_{1}(\mathbf{S}, M, N)=\operatorname{blkdiag}(\overbrace{\mathbf{S}^{(\nu)}, \mathbf{S}^{(v)}, \ldots, \mathbf{S}^{(\nu)}}^{M}), \\
& \operatorname{perm}_{2}(\mathbf{S}, M, N)=\mathbf{S}^{(\mu+\nu)} \\
& \mathbf{S}^{(\kappa)}=\operatorname{Toeplitz}\left(\left[c_{0}^{(\kappa)}, c_{1}^{(\kappa) *}, \ldots, c_{N-1}^{(\kappa) *}\right]\right), \quad \kappa=\mu, \nu, \mu+\nu,
\end{aligned}
$$

by excluding entries of $\mathbf{S}$ which are uncorrelated, and $\mathbf{s}_{m}^{(\nu)}$ and $\mathbf{s}_{n}^{(\mu) T}$ are the $m$ th column and $n$th row of $\mathbf{S}$, respectively. If the noises are IID processes where $\Sigma_{N}^{-1}$ is proportional to the identity matrix, $\operatorname{tr}\left(\Sigma_{N}^{-1} S\right)$ of (94) will be replaced by $N M[\mathbf{S}]_{1,1}$.

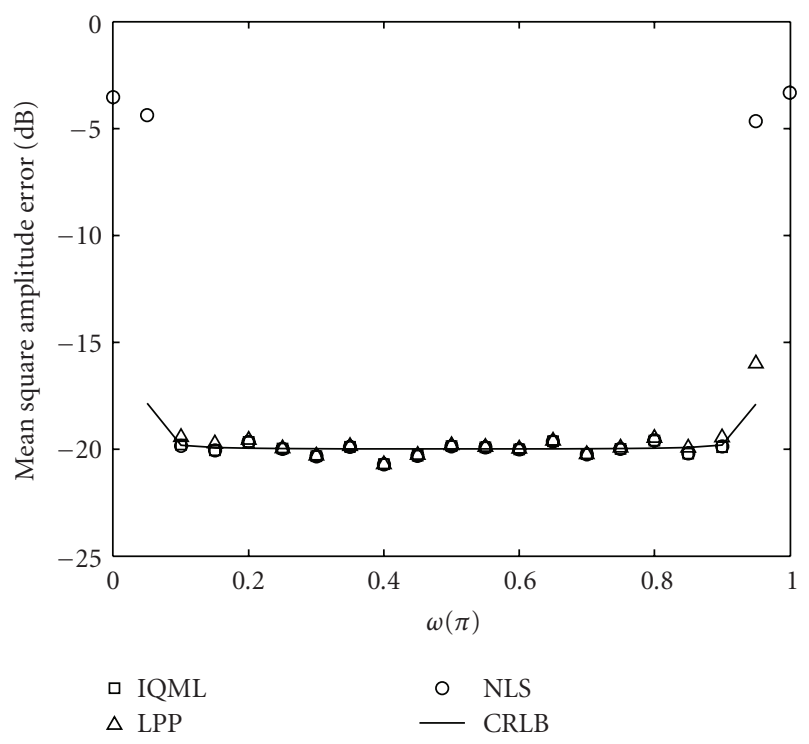

FIGURE 13: MSE for $A$ of single real sinusoid at $S N R=20 \mathrm{~dB}$.

\section{Fast Algorithm}

In the previous sections, we come across some constraints with the following structure:

$$
\left[\begin{array}{cc}
\mathbf{T} & \mathbf{t} \\
\mathbf{t}^{H} & 1
\end{array}\right] \succeq \mathbf{0}_{N+1}
$$

$$
\text { s.t. } \mathbf{T}=\operatorname{Toeplitz}\left(\left[\tau_{0}, \tau_{1}, \ldots, \tau_{N-1}\right]\right) \text {, }
$$

where $\mathbf{T}$ is a dummy matrix, $\mathbf{t}=[t(1), t(2), \ldots, t(N)]^{T}$ and $\left\{\tau_{n}\right\}$ are variables. They appear in the single complex tone models, namely, (7), (21), (84), (90), and (94). When $N$ is large, it is desirable to break down $\mathrm{T} \in \mathbb{C}^{N}$ to reduce the computational complexity. Indeed, (95) can be decomposed into

$$
\left[\begin{array}{ccc}
\tau_{0} & \tau_{i-j} & t(i) \\
\tau_{i-j}^{*} & \tau_{0} & t(j) \\
t(i)^{*} & t(j)^{*} & 1
\end{array}\right] \succeq \mathbf{0}_{3}, \quad i>j=1,2, \ldots, N,
$$

where (96) corresponds to $N(N-1) / 2$ semidefinite constraints with size $3 \times 3$ which is analogous to the ESPD [37]. As an illustration, (7) can be written as

$$
\begin{aligned}
& \max _{\{c(i)\}} \sum_{i>j}^{N} y(i-j) c(i-j)+\left(\sum_{i>j}^{N} y(i-j) c(i-j)\right)^{*} \\
& \text { s.t. }\left[\begin{array}{ccc}
1 & c(i-j) & c(i) \\
c(i-j)^{*} & 1 & c(j) \\
c(i)^{*} & c(j)^{*} & 1
\end{array}\right] \succeq \mathbf{0}_{3}, \quad i>j=1,2, \ldots, N,
\end{aligned}
$$

where $\tau_{0}=1, \tau_{n}=c(n), n=1,2, \ldots, N-1$, and $t(n)=c(n)$, $n=1,2, \ldots, N$. Furthermore, the entries of $\mathbf{C}$ are replaced with the affine objective function. 


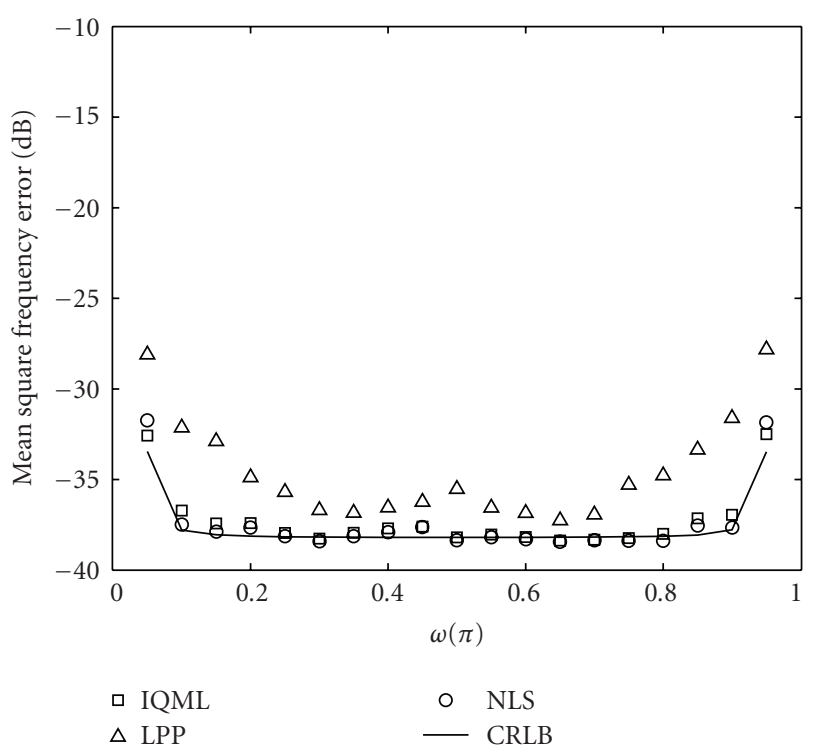

Figure 14: MSE for $\omega$ of single real sinusoid at $S N R=20 \mathrm{~dB}$.

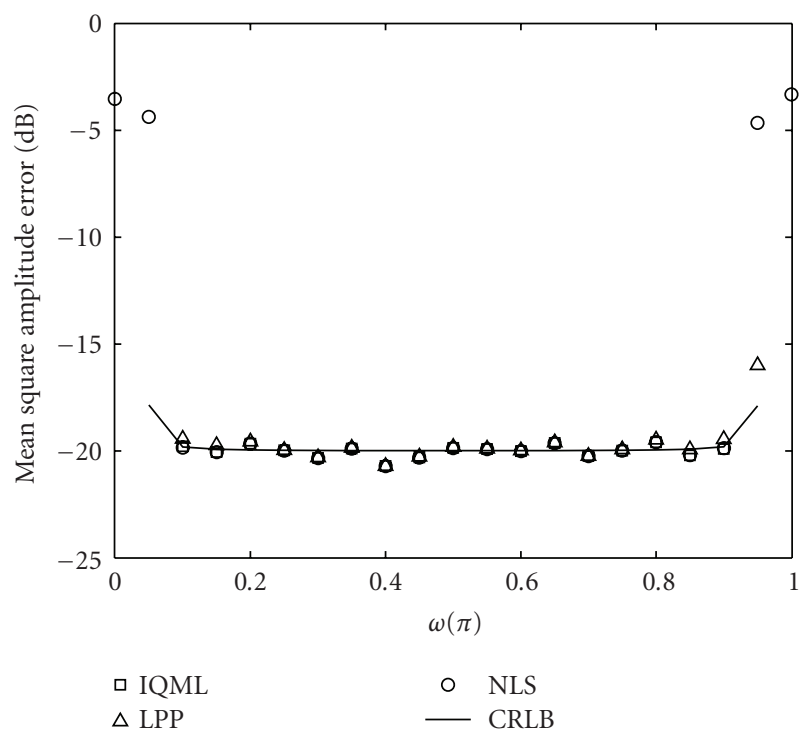

FIGURE 15: MSE for $\phi$ of single real sinusoid at $S N R=20 \mathrm{~dB}$.

\section{Simulation Results}

Computer simulations are conducted to evaluate the sinusoidal parameter estimation performance of the proposed SDR approach by comparing with the IQML estimator and CRLB in terms of mean square error (MSE). We utilize the MATLAB toolbox YALMIP [38] to realize all SDP algorithms where the solvers of SeDuMi [29] and SDPT3 [30, 31] are employed. In the IQML scheme, $\boldsymbol{\Sigma}_{e}$ is initialized by the identity matrix with $\ell=3$ iterations, and amplitude and phase estimation is performed by LS after obtaining the frequencies. The additive noises are zero-mean white complex/real Gaussian processes and they are scaled to

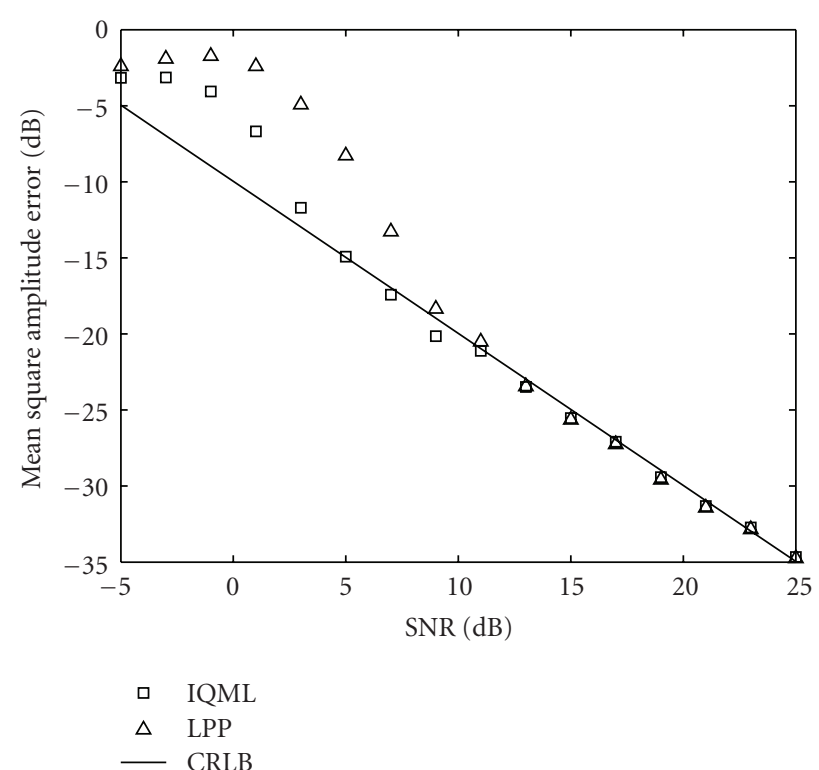

Figure 16: MSE for $A_{1}$ of multiple complex sinusoids.

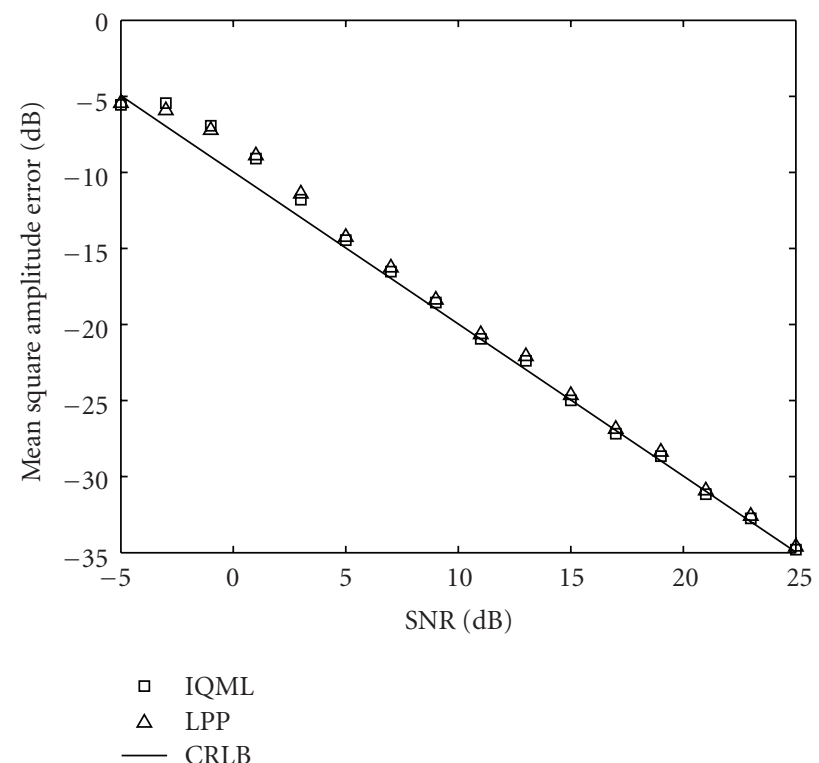

Figure 17: MSE for $A_{2}$ of multiple complex sinusoids.

obtain different signal-to-noise ratio (SNR) conditions. All results are based on 500 independent runs.

In the first test, we examine the single complex tone signal model and the sinusoidal parameters are assigned as $A=\sqrt{2}, \omega=0.6 \pi$, and $\phi=0.8 \pi$. The data length is $N=20$. Figures 1 to 3 show the MSEs for $A, \omega$, and $\phi$, respectively, using the proposed SDR methods and IQML estimator as well as CRLB. It is seen that the SDR approximations for the periodogram and NLS estimators perform almost identical and give the best performance in terms of estimation accuracy and threshold SNR. This is due to the tightness of their SDR constraints. In relaxing the NLS estimator, the 


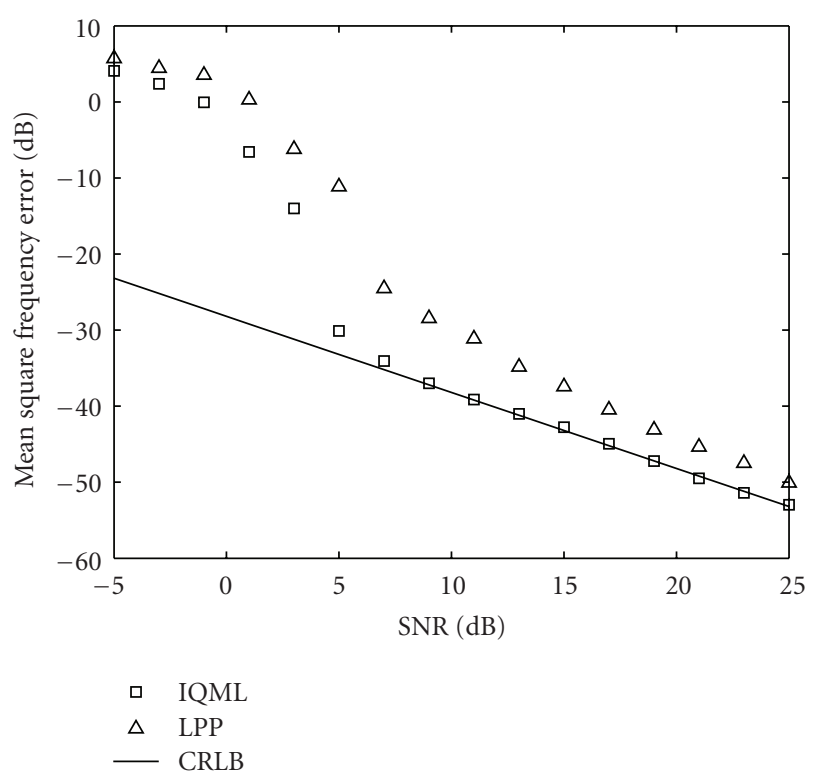

FIGURE 18: MSE for $\omega_{1}$ of multiple complex sinusoids.

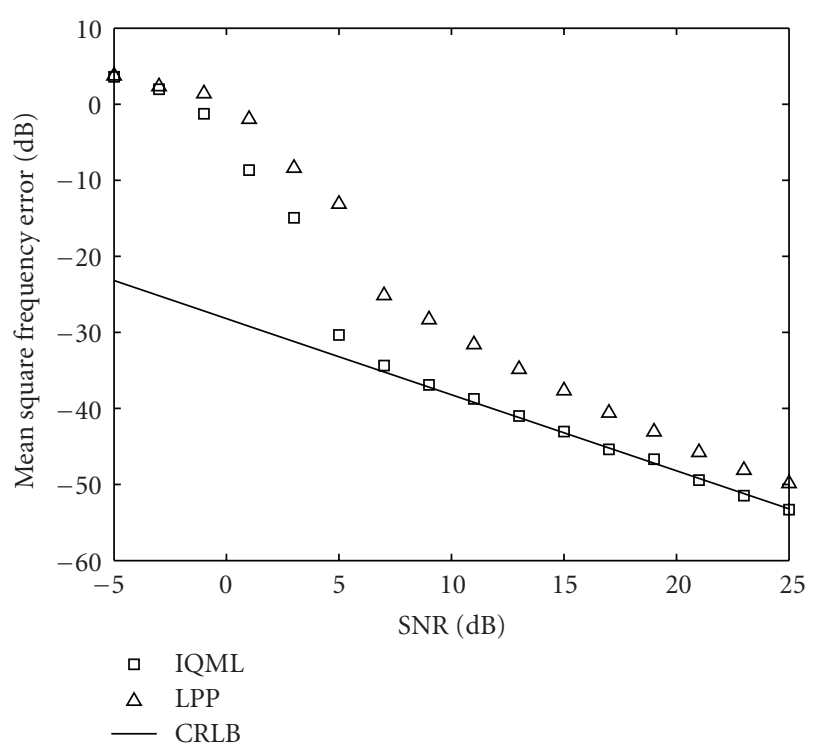

FIGURE 19: MSE for $\omega_{2}$ of multiple complex sinusoids.

differences between the lower and upper bounds are small, though equalities are replaced by positive semidefinite (PSD) constraints. While in the periodogram-based scheme, the linear constraint helps to sustain the structure even with the PSD constraints. On the other hand, the LPP and IQML methods also give optimum estimation performance but with larger threshold SNR values. They perform similarly because both relax the same cost function, although the relaxation techniques are different. Larger threshold SNRs also indicate that the relaxation in the LPP methodology is not tight enough. Regarding computational complexity of different algorithms, a comparison is provided in Table 2 . The complexity analysis of the convex algorithms is evaluated

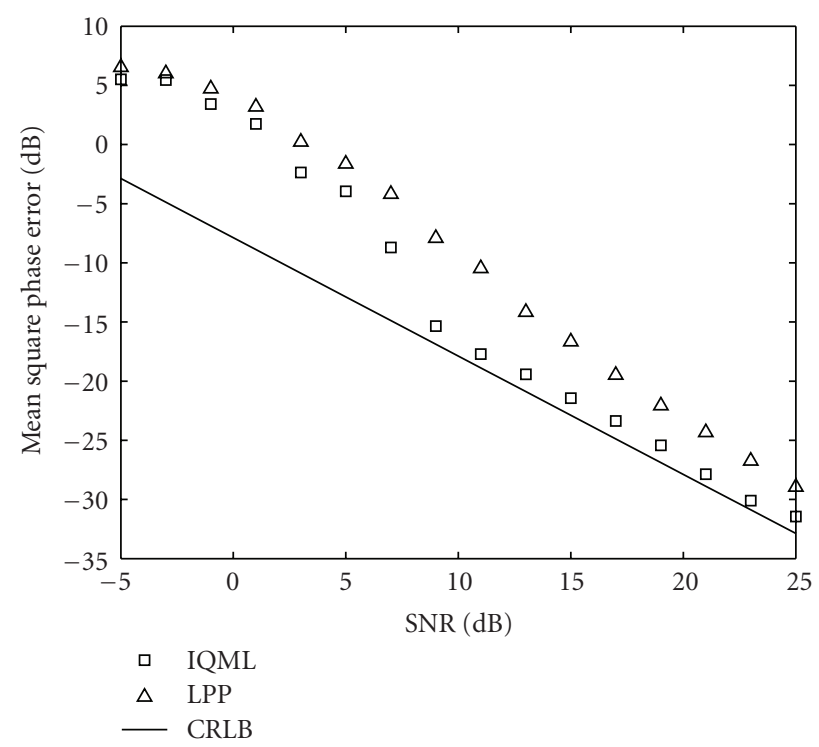

FIgURE 20: MSE for $\phi_{1}$ of multiple complex sinusoids.

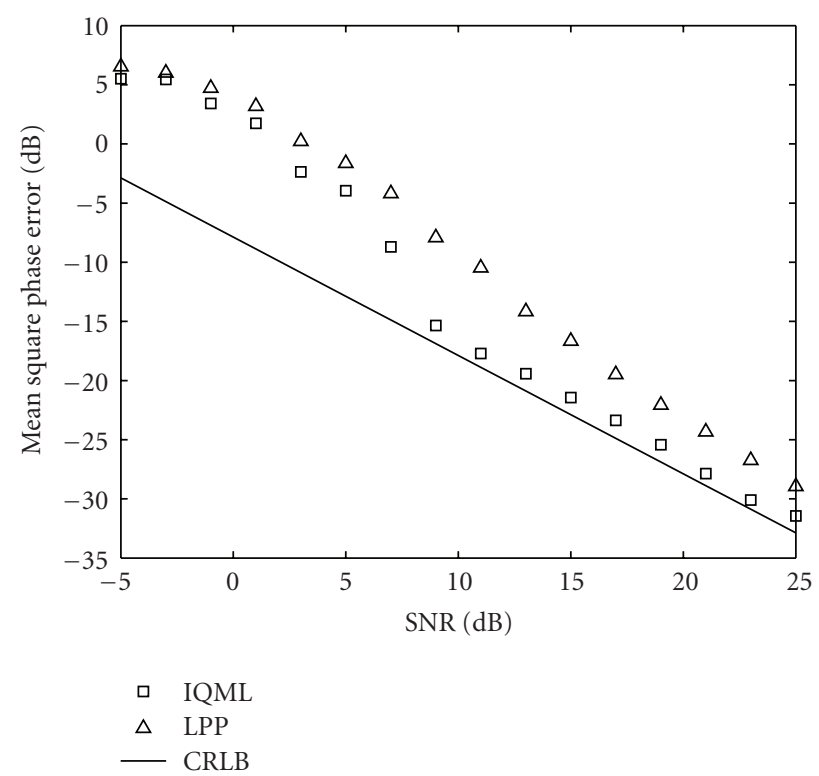

Figure 21: MSE for $\phi_{2}$ of multiple complex sinusoids.

using [17]. In summary, their operations per iteration are around $\mathcal{O}\left(N^{3}\right)$ and the required iteration number is roughly of $\mathcal{O}\left(N^{0.5}\right)$ and thus the overall complexity is $\mathcal{O}\left(N^{3.5}\right)$. On the other hand, the IQML method is less computationally demanding as its complexity is around $\mathcal{O}\left(N^{3}\right)$ because the iteration number $\ell$ is usually a small integer.

The above test is repeated with $N=128$ while all other parameters are same as before, and the results are plotted in Figures 4, 5, and 6. Basically, the findings are similar to those of Figures 1, 2, and 3, although now the threshold and/or MSE performance improves because of a larger $N$.

In the third test, parameter estimation of a real tone in white Gaussian noise is investigated. The values of the 


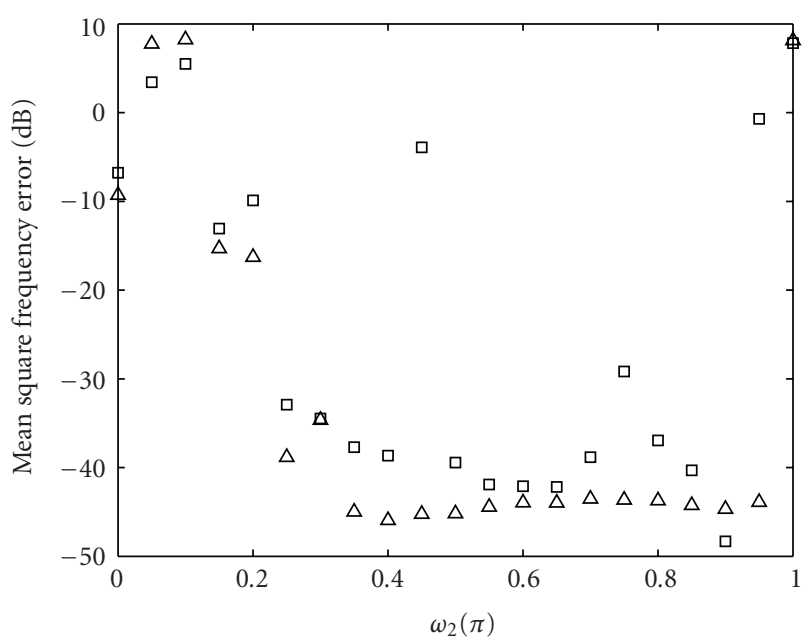

口 IQML

$\triangle$ LPP

Figure 22: MSE for $\omega_{1}$ of multiple complex sinusoids versus $\omega_{2}$.

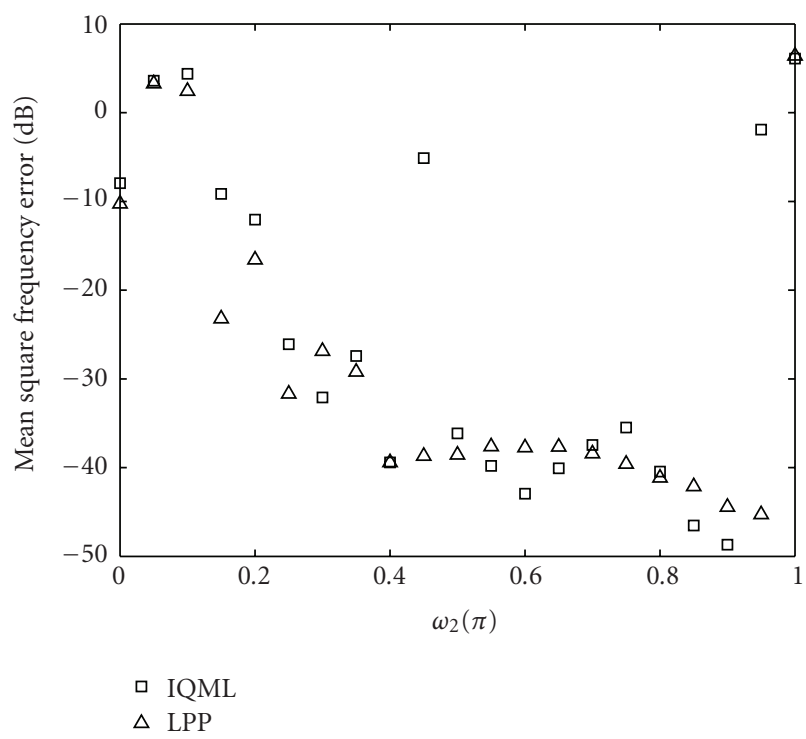

FIGURE 23: MSE for $\omega_{2}$ of multiple complex sinusoids versus $\omega_{2}$.

TABLE 2: Complexity comparison of different algorithms.

\begin{tabular}{lcc}
\hline Algorithm & Iteration number & Operations per iteration \\
\hline Periodogram & $O\left((N+1)^{1 / 2}\right)$ & $O\left((N+1)^{2}(N-1)\right)$ \\
LPP & $O\left((N)^{1 / 2}\right)$ & $O\left((N+1)^{2}(N-1)\right)$ \\
NLS & $O\left((N+1)^{1 / 2}\right)$ & $O\left((N+1)^{2} N\right)$ \\
IQML & $\ell=3$ & $O\left((N-1)^{3}\right)$ \\
\hline
\end{tabular}

sinusoidal parameters and observation length are identical to those of the first study. The MSE results for the amplitude, frequency, and phase are shown in Figures 7, 8, and 9, respectively. Again, the SDR-NLS method gives the best estimation performance with smallest threshold SNR values.

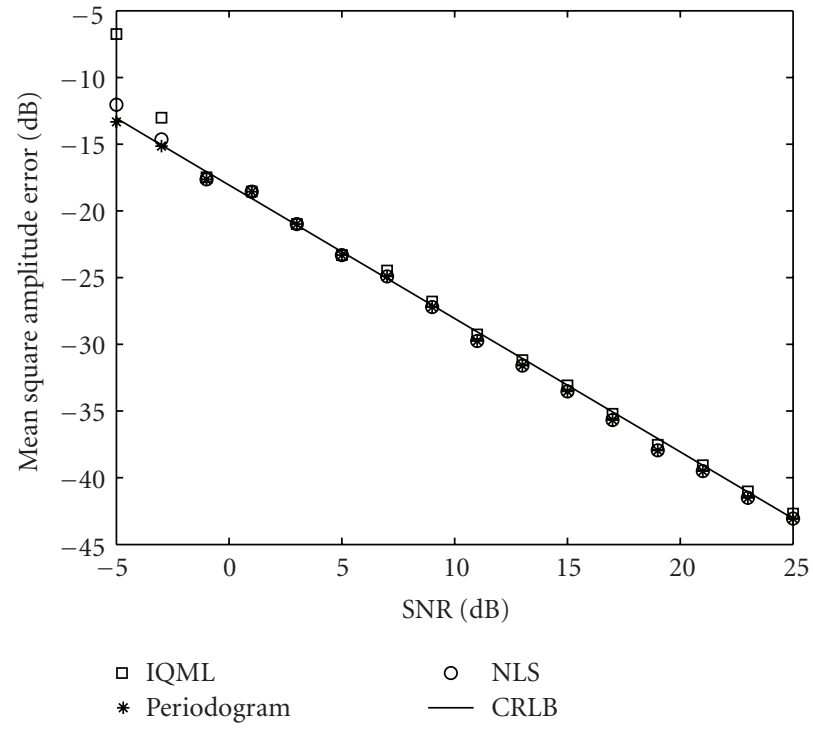

FIgure 24: MSE for $A$ of 2D single complex sinusoid.

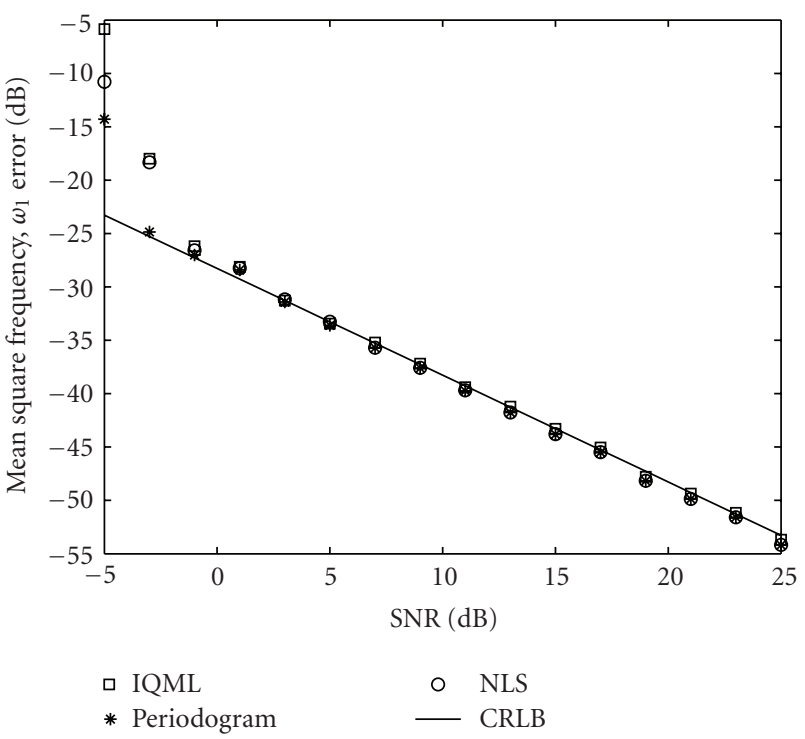

FIgURE 25: MSE for $\mu$ of 2D single complex sinusoid.

At higher SNRs, the IQML method is also an optimum estimator. Nevertheless, the SDR-LPP scheme is a suboptimal estimator as its MSEs for $\omega$ and $\phi$ cannot attain the CRLBs, although its amplitude estimation performance is optimum when $\mathrm{SNR}>5 \mathrm{~dB}$. This experiment has been repeated with a random phase model where $\phi$ is a uniform random variable between $(0,2 \pi)$ at each trial. The corresponding results are provided in Figures 10, 11, and 12 and we observe that there is no significant difference comparing with the fixed phase scenario. In Figures 13, 14, and 15, we study the MSE performance versus $\omega \in(0, \pi)$ at $\mathrm{SNR}=20 \mathrm{~dB}$ and all parameter settings are identical to the previous test. In addition to the approximate uniform estimation performance while the SDR-LPP scheme has a higher frequency dependence, similar findings are observed. 


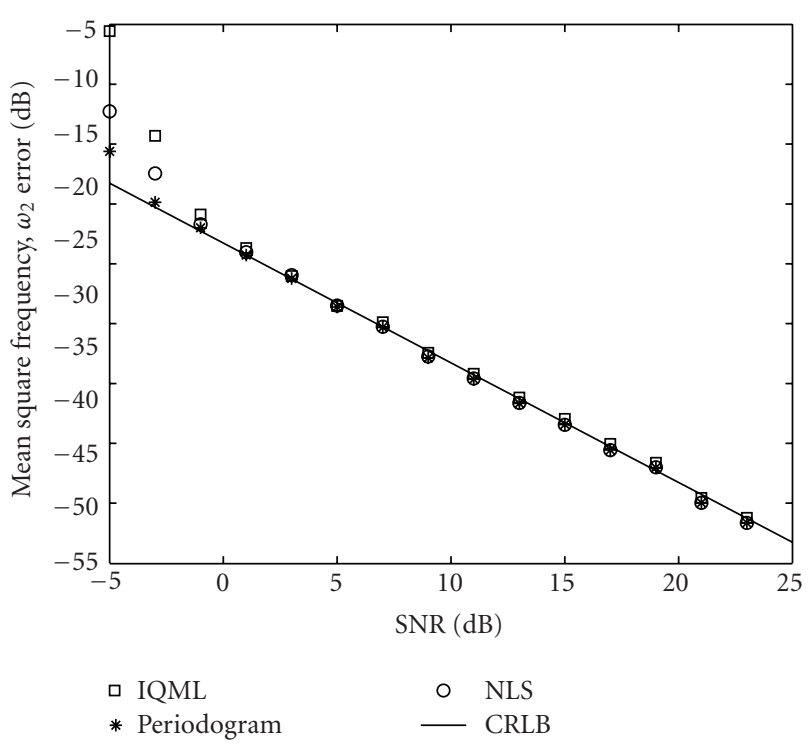

FIGURE 26: MSE for $v$ of 2D single complex sinusoid.

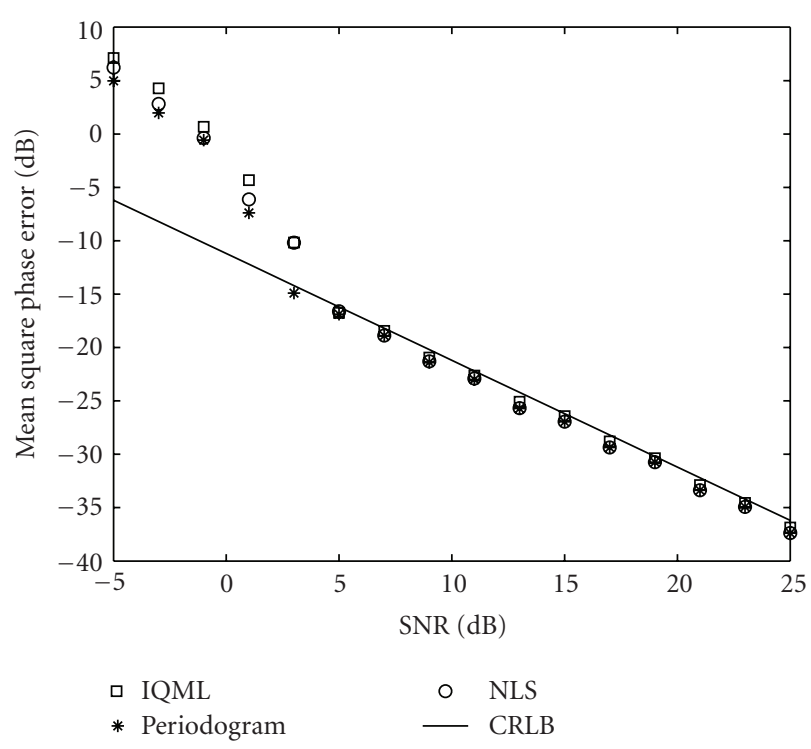

Figure 27: MSE for $\phi$ of 2D single complex sinusoid.

The multiple complex tone signal model is examined in the fourth test. We employ a dual tone with the following parameters, $A_{1}=A_{2}=\sqrt{2}, \omega_{1}=0.1 \pi, \omega_{2}=0.9 \pi$, $\phi_{1}=0.2 \pi$, and $\phi_{2}=0.8 \pi$, and $N=20$ is assigned. The MSE results of the SDR-LPP and IQML methods are plotted in Figures 16, 17, 18, 19, 20, and 21. As in the second test, the LPP scheme provides optimum amplitude estimation performance for sufficiently high SNRs but its MSEs for frequencies and phases fail to reach the CRLBs. On the other hand, the IQML method is superior to the SDR approach in frequency and phase estimation. We also study the frequency resolution issue by varying $\omega_{2}$ from 0 to $\pi$. The corresponding mean square frequency errors are shown in Figures 22 and 23. All other parameters are kept unchanged. It is seen that the SDR-LPP algorithm shows a better performance compared to IQML method when the frequencies are well separated and their performance is comparably poor for small frequency separation. Note that the MSE results for other sinusoidal parameters are not provided here as they give similar observations.

Finally, parameter estimation performance of SDR approximations for the periodogram and NLS estimators as well as the IQML method for a 2D complex tone is studied and the results are plotted in Figures 24, 25, 26, and 27. The sinusoidal parameters are $A=\sqrt{2}, \mu=0.3 \pi, \nu=0.6 \pi$, and $\phi=0.8 \pi$ with $M=N=8$. Although all three methods give optimum estimation accuracy at sufficiently large SNR conditions, the periodogram scheme has the smallest threshold SNRs, while the performance of the SDRNLS method is similar to that of the IQML approach.

\section{Conclusion}

A comprehensive study of formulating semidefinite relaxation (SDR) programs for sinusoidal parameter estimation problems has been conducted. We have proposed three common ways to relax the nonconvex maximum likelihood (ML) optimization problems regarding the periodogram, linear prediction polynomial (LPP), and nonlinear least squares (NLS) methods, to convex programs. It is shown that the SDR version of the periodogram gives the best estimation performance for a single complex sinusoid, including the two-dimensional signal model, while the SDR-NLS scheme is optimum for both the single complex and real tone models. Although the SDR-LPP methodology is flexible in the sense that it can be utilized in a wide range of ML cost functions, it only provides optimum estimation performance for a single complex sinusoid.

\section{Appendix}

In this appendix, we present the development of the permutation operators perm $1(\mathbf{S}, M, N)$ and $\operatorname{perm}_{2}(\mathbf{S}, M, N)$ via deriving the mapping of elements for $\mathbf{S}$.

For $\operatorname{perm}_{1}(\mathbf{S}, M, N)$, we aim at mapping the entries between $\operatorname{vec}(\mathbf{S}) \operatorname{vec}(\mathbf{S})^{H}$ and $\operatorname{vec}\left(\mathbf{S}^{T}\right) \operatorname{vec}\left(\mathbf{S}^{T}\right)^{H}$, so that linear constraints can be established. Mathematically, our task is to relate the indices $i, j, k$, and $l$, in the following equation

$$
\left[\operatorname{vec}(\mathbf{S}) \operatorname{vec}(\mathbf{S})^{H}\right]_{i, j}=\left[\operatorname{vec}\left(\mathbf{S}^{T}\right) \operatorname{vec}\left(\mathbf{S}^{T}\right)^{H}\right]_{k, l}
$$

First we consider the relationship between $i$ and $k$ by writing the RHS of (A.1) as:

$$
\begin{aligned}
& {\left[\operatorname{vec}(\mathbf{S}) \operatorname{vec}(\mathbf{S})^{H}\right]_{i, j}} \\
& \quad=[\operatorname{vec}(\mathbf{S})]_{i}\left[\operatorname{vec}(\mathbf{S})^{H}\right]_{j}, \quad i, j=1,2, \ldots, M N .
\end{aligned}
$$

It is clear that $[\operatorname{vec}(\mathbf{S})]_{i}$ has the form of

$$
\begin{gathered}
{[\operatorname{vec}(\mathbf{S})]_{i}=[\mathbf{S}]_{y, x}, \quad i=1,2, \ldots, M N} \\
y=1,2, \ldots, M, \quad x=1,2, \ldots, N .
\end{gathered}
$$


The indices $x$ and $y$ are related with $i$ by

$$
\begin{gathered}
y=\left\lfloor\frac{i}{N}\right\rfloor+1, \\
x=i-N\left\lfloor\frac{i}{N}\right\rfloor, \quad i=1,2, \ldots, M N,
\end{gathered}
$$

which correspond to reverse operation of vectorization. On the other hand, we have $[\mathbf{S}]_{y, x}=\left[\mathbf{S}^{T}\right]_{x, y}$ to yield

$$
\begin{array}{r}
{\left[\mathbf{S}^{T}\right]_{x, y}=\left[\operatorname{vec}\left(\mathbf{S}^{T}\right)\right]_{k}, \quad i=1,2, \ldots, M N} \\
y=1,2, \ldots, M, \quad x=1,2, \ldots, N
\end{array}
$$

with

$$
k=(x-1) M+y
$$

which corresponds to the vectorization operation. Substituting (A.4) and (A.5) into (A.7), $k$ is expressed in terms of $i$ as

$$
k=M(i-1)-(M N-1)\left\lfloor\frac{i}{N}\right\rfloor+1 .
$$

By replacing $k$ with $l$, and $i$ with $j$ in (A.8), we have

$$
l=M(j-1)-(M N-1)\left\lfloor\frac{j}{N}\right\rfloor+1 .
$$

The operator of $\operatorname{perm}_{1}(\mathbf{S}, M, N)$ is thus obtained.

For $\operatorname{perm}_{2}(\mathbf{S}, M, N)$, we aim at finding the mappings between $\{k, l\}$ and $\{i, j\}$ in

$$
\begin{aligned}
{\left[\operatorname{vec}(\mathbf{S}) \operatorname{vec}(\mathbf{S})^{H}\right]_{i, j} } & =\left[\operatorname{diag}(\mathbf{S}, 0) \operatorname{diag}(\mathbf{S}, 0)^{H}\right]_{k, l} \\
& =[\mathbf{S}]_{k, k}[\mathbf{S}]_{l, l}^{*} .
\end{aligned}
$$

Note that the valid range of $k$ and $l$ is $[1, \min ([M, N])]$, while that of $i$ and $j$ is $[1, M N]$. With the use of (A.2) and (A.10), we get

$$
\begin{gathered}
{[\operatorname{vec}(\mathbf{S})]_{i}=[\mathbf{S}]_{k, k},} \\
k=1,2, \ldots, \min ([M, N]), \\
i=1,2, \ldots, M N .
\end{gathered}
$$

It is observed that $i$ and $k$ are related by

$$
\begin{array}{r}
i=(k-1)(M+1)+1=M(k-1)+k, \\
k=1,2, \ldots, \min ([M, N]), \quad 1 \leq 1 \leq M N .
\end{array}
$$

By replacing $k$ with $l$, and $i$ with $j$ in (A.12), we obtain

$$
\begin{array}{r}
j=M(l-1)+l, \quad l=1,2, \ldots, \min ([M, N]), \\
1 \leq j \leq M N .
\end{array}
$$

This yields the operator of $\operatorname{perm}_{2}(\mathbf{S}, M, N)$.

\section{Acknowledgment}

The work described in this paper was supported by a grant from CityU (Project no. 7002499).

\section{References}

[1] S. L. Marple, Digital Spectral Analysis with Applications, Prentice-Hall, Englewood Cliffs, NJ, USA, 1987.

[2] S. M. Kay, Modern Spectral Estimation: Theory and Application, Prentice-Hall, Englewood Cliffs, NJ, USA, 1988.

[3] P. Stoica, "List of references on spectral line analysis," Signal Processing, vol. 31, no. 3, pp. 329-340, 1993.

[4] B. G. Quinn and E. J. Hannan, The Estimation and Tracking of Frequency, Cambridge University Press, Cambridge, UK, 2001.

[5] P. Stoica and R. Moses, Spectral Analysis of Signals, PrenticeHall, Upper Saddle River, NJ, USA, 2005.

[6] S. Parthasarathy and D. W. Tufts, "Maximum-likelihood estimation of parameters of exponentially damped sinusoids," Proceedings of the IEEE, vol. 73, no. 10, pp. 1528-1530, 1985.

[7] R. Kumaresan, L. L. Scharf, and A. K. Shaw, "An algorithm for pole-zero modeling and spectral analysis," IEEE Transactions on Acoustics, Speech and Signal Processing, vol. 34, no. 3, pp. 637-640, 1986.

[8] Y. Bresler and A. Macovski, "Exact maximum likelihood parameter estimation of superimposed exponential signals in noise," IEEE Transactions on Acoustics, Speech, and Signal Processing, vol. 34, no. 5, pp. 1081-1089, 1986.

[9] P. M. Djuric and S. M. Kay, "Parameter estimation of chirp signals," IEEE Transactions on Acoustics, Speech, and Signal Processing, vol. 38, no. 12, pp. 2118-2126, 1990.

[10] S. Peleg and B. Porat, "Estimation and classification of polynomial-phase signals," IEEE Transactions on Information Theory, vol. 37, no. 2, pp. 422-430, 1991.

[11] R. C. Rao, L. Zhao, and B. Zhou, "Maximum likelihood estimation of 2-D superimposed exponential signals," IEEE Transactions on Signal Processing, vol. 42, no. 7, pp. 1795-1802, 1994.

[12] P. Handel, "Properties of the IEEE-STD-1057 four-parameter sine wave fit algorithm," IEEE Transactions on Instrumentation and Measurement, vol. 49, no. 6, pp. 1189-1193, 2000.

[13] T. E. Oliphant, "On parameter estimates of the lossy wave equation," IEEE Transactions on Signal Processing, vol. 56, no. 1, pp. 49-60, 2008.

[14] P. Stoica, R. L. Moses, B. Friedlander, and T. Soderstrom, "Maximum likelihood estimation of the parameters of multiple sinusoids from noisy measurements," IEEE Transactions on Acoustics, Speech, and Signal Processing, vol. 37, no. 3, pp. 378-392, 1989.

[15] D. C. Rife and R. R. Boorstyn, "Single-tone parameter estimation from discrete-time observations," IEEE Transactions on Information Theory, vol. 20, no. 5, pp. 591-598, 1974.

[16] G. W. Lank, I. S. Reed, and G. E. Pollon, "A semicoherent detection and Doppler estimation statistic," IEEE Transactions on Aerospace and Electronic Systems, vol. 9, no. 2, pp. 151-165, 1973.

[17] L. Vandenberghe and S. Boyd, "Semidefinite programming," SIAM Review, vol. 38, no. 1, pp. 49-95, 1996

[18] S. Boyd and L. Vandenberghe, Convex Optimization, Cambridge University Press, Cambridge, UK, 2004. 
[19] Z.-Q. Luo and W. Yu, "An introduction to convex optimization for communications and signal processing," IEEE Journal on Selected Areas in Communications, vol. 24, no. 8, pp. 14261438, 2006.

[20] J. Dahl, B. H. Fleury, and L. Vandenberghe, "Approximate maximum-likelihood estimation using semidefinite programming," in Proceedings of the IEEE International Conference on Acoustics, Speech, and Signal Processing (ICASSP '03), vol. 6, pp. 721-724, Montreal, Canada, May 2003.

[21] Y. Yang, C. Zhao, P. Zhou, and W. Xu, "MIMO detection of 16QAM signaling based on semidefinite relaxation," IEEE Signal Processing Letters, vol. 14, no. 11, pp. 797-800, 2007.

[22] M. Nekuii, M. Kisialiou, T. N. Davidson, and Z.-Q. Luo, "Efficient soft demodulation of MIMO QPSK via semidefinite relaxation," in Proceedings of the IEEE International Conference on Acoustics, Speech, and Signal Processing (ICASSP '08), pp. 2665-2668, Las Vegas, Nev, USA, March 2008.

[23] W.-K. Ma, C.-C. Su, J. Jaldén, and C.-Y. Chi, "Some results on 16-QAM MIMO detection using semidefinite relaxation," in Proceedings of the IEEE International Conference on Acoustics, Speech, and Signal Processing (ICASSP '08), pp. 2673-2676, Las Vegas, Nev, USA, March 2008.

[24] T. Roh, B. Dumitrescu, and L. Vandenberghe, "Multidimensional FIR filter design via trigonometric sum-of-squares optimization," IEEE Journal on Selected Topics in Signal Processing, vol. 1, no. 4, pp. 641-650, 2007.

[25] T.-H. Chang, Z.-Q. Luo, and C.-Y. Chi, "Approximation bounds for semidefinite relaxation of max-min-fair multicast transmit beamforming problem," IEEE Transactions on Signal Processing, vol. 56, no. 8, pp. 3932-3943, 2008.

[26] K. W. Cheung, W.-K. Ma, and H. C. So, "Accurate approximation algorithm for TOA-based maximum likelihood mobile location using semidefinite programming," in Proceedings of the IEEE International Conference on Acoustics, Speech, and Signal Processing (ICASSP '04), vol. 2, pp. 145-148, Montreal, Canada, May 2004.

[27] P. Biswas, T.-C. Lian, T.-C. Wang, and Y. Ye, "Semidefinite programming based algorithms for sensor network localization," ACM Transactions on Sensor Networks, vol. 2, no. 2, pp. 188220, 2006.

[28] P. Biswas, T.-C. Liang, K.-C. Toh, Y. Ye, and T.-C. Wang, "Semidefinite programming approaches for sensor network localization with noisy distance measurements," IEEE Transactions on Automation Science and Engineering, vol. 3, no. 4, pp. 360-371, 2006.

[29] J. F. Sturm, "Using SeDuMi 1.02, a MATLAB toolbox for optimization over symmetric cones," Optimization Methods and Software, vol. 11, no. 1, pp. 625-653, 1999.

[30] K. C. Toh, M. J. Todd, and R. H. Tütüncü, "SDPT3-a MATLAB software package for semidefinite programming, version 1.3," Optimization Methods and Software, vol. 11, no. 1, pp. 545-581, 1999.

[31] R. H. Tütüncü, K. C. Toh, and M. J. Todd, "Solving semidefinite-quadratic-linear programs using SDPT3," Mathematical Programming, vol. 95, no. 2, pp. 189-217, 2003.

[32] Y. Nesterov and A. Nemirovskii, Interior Point Polynomial Methods in Convex Programming, SIAM, Philadelphia, Pa, USA, 1994.

[33] H. Wolkowicz, R. Saigal, and L. Vandenberghe, Handbook of Semidefinite Programing: Theory, Algorithms and Applications, Kluwer Academic Publishers, Norwell, Mass, USA, 1999.
[34] M. Aoki and P. C. Yue, "On a priori error estimates of some identification methods," IEEE Transactions on Automatic Control, vol. 15, no. 5, pp. 541-548, 1970.

[35] B. G. Quinn and J. M. Fernandes, "A fast efficient technique for the estimation of frequency," Biometrika, vol. 78, pp. 489-498, 1991.

[36] B. G. Quinn, "Recent advances in rapid frequency estimation," Digital Signal Processing, vol. 19, no. 6, pp. 942-948, 2009.

[37] Z. Wang, S. Zheng, Y. Ye, and S. Boyd, "Further relaxations of the semidefinite programming approach to sensor network localization," SIAM Journal on Optimization, vol. 19, no. 2, pp. 655-673, 2008.

[38] J. Löfberg, "YALMIP: a toolbox for modeling and optimization in MATLAB," in Proceedings of the IEEE International Symposium on Computer-Aided Control System Design (ACSD '04), pp. 284-289, Taipei, Taiwan, September 2004. 